\title{
Extended virtual element method for two-dimensional linear elastic fracture
}

\author{
E. Benvenuti ${ }^{\mathrm{a}}$, A. Chiozzi ${ }^{\mathrm{a}, *}$, G. Manzini ${ }^{\mathrm{b}}$, N. Sukumar ${ }^{\mathrm{c}}$ \\ ${ }^{a}$ Department of Engineering, University of Ferrara, Via Saragat 1, 44122 Ferrara, Italy \\ ${ }^{b}$ Istituto di Matematica Applicata e Tecnologie Informatiche, Consiglio Nazionale delle Ricerche, Pavia, Italy, \\ ${ }^{c}$ Department of Civil and Environmental Engineering, University of California, Davis, CA 95616, USA
}

\begin{abstract}
In this paper, we propose an eXtended Virtual Element Method (X-VEM) for two-dimensional linear elastic fracture. This approach, which is an extension of the standard Virtual Element Method (VEM), facilitates mesh-independent modeling of crack discontinuities and elastic crack-tip singularities on general polygonal meshes. For elastic fracture in the X-VEM, the standard virtual element space is augmented by additional basis functions that are constructed by multiplying standard virtual basis functions by suitable enrichment fields, such as asymptotic mixed-mode crack-tip solutions. The design of the X-VEM requires an extended projector that maps functions lying in the extended virtual element space onto a set spanned by linear polynomials and the enrichment fields. An efficient scheme to compute the mixed-mode stress intensity factors using the domain form of the interaction integral is described. The formulation permits integration of weakly singular functions to be performed over the boundary edges of the element. Numerical experiments are conducted on benchmark mixed-mode linear elastic fracture problems that demonstrate the sound accuracy and optimal convergence in energy of the proposed formulation.
\end{abstract}

Keywords: partition-of-unity enrichment; X-VEM; crack discontinuity; crack-tip singularity; mixed-mode fracture; polygonal meshes

\section{Introduction}

Over the past two decades, significant attention has been devoted to the development of numerical techniques to solve problems that admit singular or discontinuous solutions such as fracture propagation in solids. Among these techniques, enriched finite element approximations based on the partition-of-unity framework [1, 2] have received considerable attention. The eXtended Finite Element Method (X-FEM) [3] is one of the most successful methods to analyse fracture problems on unstructured triangular and quadrilateral meshes without requiring remeshing. For fracture simulations on polygonal meshes, extended finite element formulations have been proposed [4, 5] as well as the scaled boundary element method [6-8]. However,

\footnotetext{
* Corresponding author

Email addresses: elena. benvenuti@unife.it (E. Benvenuti), andrea.chiozzi@unife.it (A. Chiozzi), marco.manzini@imati.cnr.it (G. Manzini), nsukumar@ucdavis.edu (N. Sukumar)
} 
construction of shape functions that are defined on general polygons renders extended finite element formulations to be more involved and numerical integration of regular and weakly singular functions over polygons is also an issue that requires special attention [9-11].

The Virtual Element Method (VEM) [12] is a stabilized Galerkin formulation to solve partial differential equations on very general polygonal meshes that overcomes the many difficulties and challenges that are associated with polygonal finite element formulations. The VEM derives from the mimetic finite difference method [13, 14] and is a generalization of the Finite Element Method (FEM) in which the explicit knowledge of the basis functions is not needed. Such basis functions are defined as the solution of a local elliptic partial differential equation, and are never explicitly computed in the implementation of the method. Indeed, the VEM uses the elliptic projections of the basis functions onto suitable polynomial spaces to discretize the bilinear form and the continuous linear functional deriving from the variational formulation. Such projections are computable because of a careful choice of the degrees of freedom. The discretized bilinear form is conveniently decomposed as the sum of a consistent term, which ensures polynomial consistency, and a correction term, which guarantees stability. Moreover, the VEM requires the same element-wise assembly procedure of the FEM for the construction of the global stiffness matrix, thus resulting in a linear system of equations from which the solution is obtained.

In recent years, the VEM has also been used to solve problems in solid mechanics, such as two- and three-dimensional linear elasticity [15, 16], nearly incompressible elasticity [17,-19], inelastic problems [20, 21], mixed variational formulations for linear elasticity [22, 23], linear elasticity on curvilinear elements [24], and elastodynamics [25-27]. However, very few studies have exploited the flexibility of the method to deal with meshes that are cut by discontinuities and/or contain interior singularities. Among these we mention the virtual element modeling of flow in fracture networks [28] and the application of the VEM to 2D elastic fracture problems [29-31]. In these studies, hanging nodes are inserted at locations where each discontinuity intersects an element, so that each cut element is partitioned into a collection of polygonal elements.

Approximating spaces that consist of the product of low-order virtual element basis functions and a nonpolynomial function were first proposed in [32] for the Helmholtz problem, where the nonpolynomial function is chosen as a planewave in the two directions. More recently, drawing inspiration from the X-FEM, an eXtended Virtual Element Method (X-VEM) is presented in [33] to treat singularities and crack discontinuities in the scalar Laplace problem, which also governs the deformation of a stretched membrane or torsion in a prismatic beam [34]. An enriched nonconforming virtual element method is proposed in [35], where the approximation spaces is enriched with special singular functions (without using the partitionof-unity framework) to solve the Poisson problem with reentrant corners.

In this paper, we develop an extended virtual element formulation for linear elastic fracture problems, in which the displacement field features both discontinuities and crack-tip singularities. For the X-VEM, we construct an enriched virtual element space by introducing an additional set of virtual basis functions, which are built on vectorial enrichment fields that are suitably chosen so that they reproduce the nature of the weak singularity in the neighborhood of the crack tip. Hence, additional information about the exact solution is incorporated in the computational method, mitigating the effects of the singularity on the numerical accuracy. In principle, any number of auxiliary fields can be considered to enrich the virtual element space. In the X-FEM, near-tip crack functions are used as enrichment functions in the discrete space [3], 
whereas in the X-VEM we require the enriched stress fields to be divergence-free and hence choose the asymptotic mode I and mode II crack-tip displacement solutions as vectorial enrichments. The use of vectorial enrichments was first proposed in the generalized finite element method [36]. Furthermore, as introduced in Benvenuti et al. [33], discontinuities in the displacement field are incorporated in the virtual element space using the approach proposed for finite elements by Hansbo and Hansbo [37]. In contrast to the X-FEM, the X-VEM for elastic fracture provides greater flexibility since it is applicable to arbitrary (simple and nonsimple) polygonal meshes. Furthermore, unlike the X-FEM where special integration schemes [10] are needed to accurately evaluate the weak form (domain) integrals, in the X-VEM a one-dimensional quadrature rule on the boundary of the polygonal element suffices to compute such integrals. As in the VEM, the explicit knowledge of virtual shape functions on general polygons is not required, and as we will detail, in this particular instance of the X-VEM, weak form integrals are computed only on the boundary of the element, where the virtual shape functions are known.

The remainder of this article is organized as follows. In Section 2, we introduce the strong and weak forms for two-dimensional linear elastic fracture problems. In Section 3, we describe the extended virtual element formulation. For crack tip singularities, we devise an extended projector that maps functions that lie in the extended virtual element space onto the space spanned by the basis of linear polynomials and the enrichment fields. The approach of Hansbo and Hansbo [37] is used to model crack discontinuities in the X-VEM. The implementation of the $\mathrm{X}-\mathrm{VEM}$ is discussed in Section 4. In Section 5, we presents results for the discontinuous and extended patch tests, and show that the method delivers optimal rate of convergence in energy for benchmark mixed-mode crack problems.

Final remarks and suggestions for future work are discussed in Section 6 .

\section{Governing equations for 2D linear elasticity}

We consider a linear elastic body occupying the two-dimensional domain $\Omega \subset \mathbb{R}^{2}$, bounded by $\Gamma$ (see Fig. 11). We denote the displacement field on $\Omega$ by $\boldsymbol{u}(\boldsymbol{x})$ and assume small strains and displacements. The boundary $\Gamma=\Gamma_{u} \cup \Gamma_{t} \cup \Gamma_{c}$, where $\Gamma_{u}, \Gamma_{t}$ and $\Gamma_{c}$ are nonoverlapping, i.e., $\Gamma_{u} \cap \Gamma_{t} \cap \Gamma_{c}=\varnothing$.

Prescribed displacements $g \in C^{0}\left(\Gamma_{u}\right)$ are imposed on $\Gamma_{u}$, whereas tractions $\overline{\boldsymbol{t}} \in C^{0}\left(\Gamma_{t}\right)$ are imposed on $\Gamma_{t}$. Here, $\Gamma_{c}$ represents a traction-free internal crack.

We now summarize the governing equations of the elastic problem under the assumptions of small strains and displacements. Let $\sigma$ be the Cauchy stress tensor. In the absence of body forces, the equilibrium equations are

$$
\nabla \cdot \sigma=\mathbf{0} \quad \text { in } \Omega
$$

with the natural boundary conditions

$$
\begin{array}{ll}
\boldsymbol{\sigma} \cdot \boldsymbol{n}=\overline{\boldsymbol{t}} & \text { on } \Gamma_{t}, \\
\boldsymbol{\sigma} \cdot \boldsymbol{n}=\mathbf{0} & \text { on } \Gamma_{c},
\end{array}
$$

where $\boldsymbol{n}$ is the unit outward normal, and the essential boundary condition

$$
\boldsymbol{u}=\boldsymbol{g} \quad \text { on } \Gamma_{u} \text {. }
$$




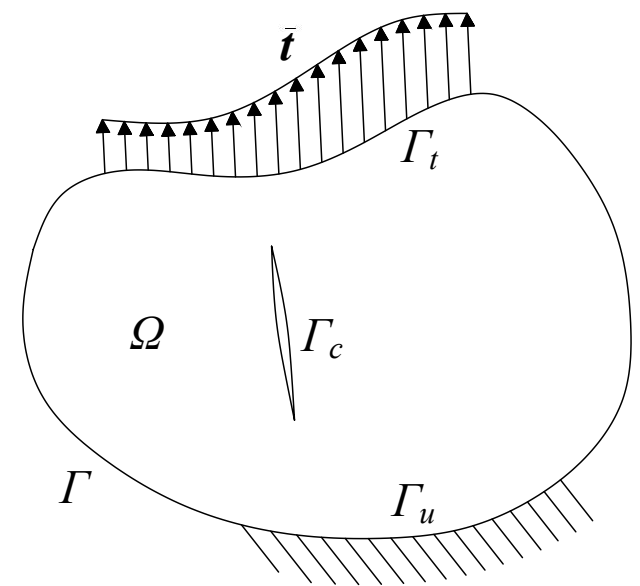

Figure 1: Elastostatic boundary-value problem for an embedded crack.

The small strain tensor $\boldsymbol{\varepsilon}$ is related to the displacement field $\boldsymbol{u}$ by the compatibility equation

$$
\boldsymbol{\varepsilon}(\boldsymbol{u})=\nabla_{s} \boldsymbol{u},
$$

where $\nabla_{s}$ is the symmetric part of the gradient operator, which is defined as

$$
\nabla_{s}(\cdot)=\frac{1}{2}\left(\nabla(\cdot)+\nabla^{T}(\cdot)\right)
$$

Lastly, the isotropic linear elastic constitutive law is

$$
\sigma(\boldsymbol{u})=\boldsymbol{C}: \boldsymbol{\varepsilon}(\boldsymbol{u})
$$

where $\boldsymbol{C}$ is the fourth-order elasticity tensor for a homogeneous isotropic material.

The weak form of the problem is constructed by defining the space of admissible displacement fields as

$$
\mathscr{U}=\left\{\boldsymbol{v} \in\left[H^{1}(\Omega)\right]^{2}: \boldsymbol{v}=\boldsymbol{g} \text { on } \Gamma_{u}, \boldsymbol{v} \text { discontinuous on } \Gamma_{c}\right\},
$$

where the space $\mathscr{V}$ is related to the regularity of the solution, and admits discontinuous functions across the crack. Similarly, the test function space is defined as:

$$
\mathscr{U}_{0}=\left\{v \in\left[H^{1}(\Omega)\right]^{2}: v=0 \text { on } \Gamma_{u}, v \text { discontinuous on } \Gamma_{c}\right\} .
$$

The weak form of the equilibrium equation reads as: Find $\boldsymbol{u} \in \mathscr{U}$ such that

$$
a(\boldsymbol{u}, \boldsymbol{v}):=\int_{\Omega} \boldsymbol{\sigma}(\boldsymbol{v}): \boldsymbol{\varepsilon}(\boldsymbol{u}) d \boldsymbol{x}=\int_{\Gamma_{t}} \overline{\boldsymbol{t}} \cdot \boldsymbol{v} d \Gamma=: b(\boldsymbol{v}) \quad \forall \boldsymbol{v} \in \mathscr{U}_{0} .
$$

The above statement is equivalent to the strong form (1a) and in a finite element framework it is solved approximately on a sequence of appropriately nested finite-dimensional subspaces of $\mathscr{U}$. 


\section{Extended virtual element formulation}

We now discuss the formulation of the extended virtual element method for two-dimensional elasticity problems. We start, in Section 3.1, from the definition and regularity properties of the mesh families for the X-VEM, and after reviewing the 'nonenriched' VEM in Section 3.2, we provide the design of the X-VEM for full and partial local enrichments in Sections 3.3 and 3.4 .

\subsection{Mesh definition and regularity assumptions}

Let $\mathcal{T}=\left\{\Omega_{h}\right\}_{h}$ be a family of decompositions of $\Omega$ into nonoverlapping polygonal elements $E$ with nonintersecting boundary $\partial E$, barycenter $\boldsymbol{x}_{E} \equiv\left(x_{E}, y_{E}\right)^{T}$, area $|E|$, and diameter $h_{E}=$ $\sup _{\boldsymbol{x}, \boldsymbol{y} \in E}|\boldsymbol{x}-\boldsymbol{y}|$. The subindex $h$ that labels each mesh $\Omega_{h}$ is the maximum of the diameters $h_{E}$ of the elements of that mesh. The boundary of $E$ is formed by $N_{E}$ straight edges connecting $N_{E}$ vertices. The sequence of the vertices on $\partial E$ is oriented in the counter-clockwise order and the vertex coordinates are denoted by $\boldsymbol{x}_{i} \equiv\left(x_{i}, y_{i}\right)^{T}, i=1,2, \ldots, N_{E}$. We denote the unit normal vector to $\partial E$ pointing out of $E$ by $\boldsymbol{n}_{E}$.

Usually, in the convergence analysis of the conforming VEM, it is assumed that there exists a positive constant $\varrho$ independent of $h$ (hence, also of $\Omega_{h}$ ) such that for every polygonal element $E \in \Omega_{h}$ it holds that:

(i) $E$ is star-shaped with respect to a disk with radius greater than $\varrho h_{E}$;

(ii) for every edge $e \in \partial E$ it holds that $h_{e} \geq \varrho h_{E}$.

Although the convergence analysis of the X-VEM is beyond the scope of this paper, we present such mesh regularity assumptions to characterize the geometry of the elements in the polygonal meshes, which is pertinent to our formulation. We also note that condition (i) implies that all the mesh elements have a finite number of vertices and edges for $h \rightarrow 0$ and are simply connected subset of $\mathbb{R}^{2}$. In turn, condition (ii) excludes the possibility of collapsing vertices in the refinement process, i.e., vertices whose distance becomes zero faster than $h$.

\subsection{Conforming virtual element space, elliptic projection and bilinear form}

Let $\Gamma_{c}=\emptyset$. On every polygonal element $E$ with boundary $\partial E$, we first define the following scalar virtual element space

$$
\left.V^{h}(E) \equiv\left\{v^{h} \in H^{1}(E): \Delta v^{h}=0, v^{h}{ }_{\mid \partial E} \in C^{0}(\partial E), v_{\mid e}^{h} \in \mathbb{P}^{1}(e) \forall e \in \partial E\right)\right\},
$$

where $\mathbb{P}^{1}(e)$ is the set of linear polynomials on the element edge $e \in \partial E$ and $\Delta$ is the Laplace operator. We denote the canonical basis of $V^{h}(E)$ by $\left\{\varphi_{i}\right\}_{i=1}^{N_{E}}$, so that each $\varphi_{i}$ is the harmonic function on $E$ with continuous piecewise linear trace on the boundary $\partial E$ that takes value 1 on the $i$-th node and 0 on the remaining nodes. The linear polynomials $\mathbb{P}^{1}(E)$ are a subspace of $V^{h}(E)$, and the basis functions $\varphi_{i}$ satisfies the partition-of-unity property

$$
\sum_{i=1}^{N_{E}} \varphi_{i}(\boldsymbol{x})=1 \quad \forall \boldsymbol{x} \in E .
$$

For the linear elasticity (vectorial) problem, on every polygonal element $E \in \Omega_{h}$ we define the local virtual element space of vector-valued functions as $\boldsymbol{V}^{h}(E)=\left[V^{h}(E)\right]^{2}$. Every vectorvalued virtual element function $\boldsymbol{v}^{h} \in \boldsymbol{V}^{h}(E)$ is uniquely characterized by its vertex values, also 
known as the degrees of freedom (DOFs) of the method. In the framework of two-dimensional elasticity, such degrees of freedom represent the two components of the displacement field at the mesh vertices. Therefore, we have $2 N_{E}$ degrees of freedom per mesh element $E$. Such degrees of freedom are unisolvent in $V^{h}(E)$ [15].

We define the set of 'canonical' basis functions of $\boldsymbol{V}^{h}(E)$ by $\left\{\boldsymbol{\varphi}_{i}\right\}_{i=1}^{2 N_{E}}$ so that $\boldsymbol{\varphi}_{2 i-1}=\left(\varphi_{i}, 0\right)^{T}$ and $\phi_{2 i}=\left(0, \varphi_{i}\right)^{T}$ for $i=1, \ldots, N_{E}$. These functions are made explicit by the following expression

$$
\boldsymbol{V}^{h}(E)=\operatorname{span}\left\{\left(\begin{array}{c}
\varphi_{1} \\
0
\end{array}\right),\left(\begin{array}{c}
0 \\
\varphi_{1}
\end{array}\right), \ldots,\left(\begin{array}{c}
\varphi_{i} \\
0
\end{array}\right),\left(\begin{array}{c}
0 \\
\varphi_{i}
\end{array}\right), \ldots,\left(\begin{array}{c}
\varphi_{N_{E}} \\
0
\end{array}\right),\left(\begin{array}{c}
0 \\
\varphi_{N_{E}}
\end{array}\right)\right\}
$$

and the partition-of-unity property (6) implies that

$$
\sum_{i=1}^{N_{E}} \boldsymbol{\varphi}_{2 i-1}(\boldsymbol{x})=\left(\begin{array}{c}
\sum_{i=1}^{N_{E}} \varphi_{i}(\boldsymbol{x}) \\
0
\end{array}\right)=\left(\begin{array}{l}
1 \\
0
\end{array}\right) \quad \text { and } \quad \sum_{i=1}^{N_{E}} \boldsymbol{\varphi}_{2 i}(\boldsymbol{x})=\left(\begin{array}{c}
0 \\
\sum_{i=1}^{N_{E}} \varphi_{i}(\boldsymbol{x})
\end{array}\right)=\left(\begin{array}{l}
0 \\
1
\end{array}\right) \quad \forall \boldsymbol{x} \in E
$$

We collect all the element spaces $V^{h}(E)$ in a conforming way and define the global virtual element space $\boldsymbol{V}^{h} \subset \mathscr{U}_{0}$ as follows

$$
\boldsymbol{V}^{h}=\left\{\boldsymbol{v}^{h} \in\left[H^{1}(\Omega)\right]^{2}: \boldsymbol{v}_{\mid E}^{h} \in \boldsymbol{V}^{h}(E) \forall E \in \Omega_{h}\right\} .
$$

Let $a^{h}(\cdot, \cdot)$ and $b^{h}(\cdot)$ denote computable counterparts of the exact bilinear form $a(\cdot, \cdot)$ and the linear functional $b(\cdot)$ acting on $\boldsymbol{V}^{h}$, and consider the virtual element affine subspace of $\boldsymbol{V}^{h}$ given by

$$
\boldsymbol{V}_{\boldsymbol{g}}^{h}=\left\{\boldsymbol{v}^{h} \in \boldsymbol{V}^{h}: \boldsymbol{v}^{h}=\boldsymbol{g}^{h} \text { on } \Gamma_{u}\right\},
$$

which incorporates the essential boundary condition $(1 \mathrm{~d})$ in the space definition by taking the linear interpolant $\boldsymbol{g}^{h}$ of $\boldsymbol{g}$, and the linear subspace $\boldsymbol{V}_{\mathbf{0}}^{h} \subset \boldsymbol{V}^{h}$ that is obtained by setting $\boldsymbol{g}^{h}=\mathbf{0}$ in $V_{g}^{h}$. With this caveat, the virtual element approximation of the variational problem (4) reads as: Find $\boldsymbol{u}^{h} \in \boldsymbol{V}_{g}^{h}$ such that

$$
a^{h}\left(\boldsymbol{u}^{h}, \boldsymbol{v}^{h}\right)=b^{h}\left(\boldsymbol{v}^{h}\right) \quad \forall \boldsymbol{v}^{h} \in \boldsymbol{V}_{\mathbf{0}}^{h} .
$$

To construct the bilinear form $a^{h}(\cdot, \cdot)$ and the linear functional $b^{h}(\cdot)$, we first split them as the sum of element terms $a^{h, E}(\cdot, \cdot)$ and $b^{h, E}(\cdot)$ so that

$$
\begin{aligned}
a^{h}\left(\boldsymbol{u}^{h}, \boldsymbol{v}^{h}\right) & =\sum_{E \in \Omega} a^{h, E}\left(\boldsymbol{u}^{h}, \boldsymbol{v}^{h}\right) \quad \forall \boldsymbol{u}^{h}, \boldsymbol{v}^{h} \in \boldsymbol{V}^{h}, \\
b^{h}\left(\boldsymbol{u}^{h}\right) & =\sum_{E \in \Omega} b^{h, E}\left(\boldsymbol{v}^{h}\right) \quad \forall \boldsymbol{v}^{h} \in \boldsymbol{V}^{h} .
\end{aligned}
$$

It is well established in the VEM literature that a crucial requirement for every $a^{h, E}(\cdot, \cdot)$ to deliver an accurate and stable formulation is to satisfy the properties of linear consistency and stability [12]. To construct such $a^{h, E}(\cdot, \cdot)$, we resort to the elliptic projection operator $\Pi^{a}: \boldsymbol{V}^{h}(E) \rightarrow\left[\mathbb{P}^{1}(E)\right]^{2}$, which maps vector-valued functions from $\boldsymbol{V}^{h}(E)$ onto linear vector 
polynomials. To fix the nontrivial kernel in the definition of such elliptic projector, we introduce the average translation operator over the $N_{E}$ element vertices $\left\{\boldsymbol{x}_{j}\right\}_{j=1}^{N_{E}}$ defined as

$$
\overline{\boldsymbol{w}}=\frac{1}{N_{E}} \sum_{j=1}^{N_{E}} \boldsymbol{w}\left(\boldsymbol{x}_{j}\right)
$$

and the average rotation operator defined as

$$
\overline{(\boldsymbol{w})}_{R}=\frac{1}{N_{E}} \sum_{j=1}^{N_{E}} \boldsymbol{r}\left(\boldsymbol{x}_{j}\right) \cdot \boldsymbol{w}\left(\boldsymbol{x}_{j}\right), \quad \boldsymbol{r}(\boldsymbol{x})=(y,-x)^{T} .
$$

For each $\boldsymbol{v}^{h} \in \boldsymbol{V}^{h}(E)$, the elliptic projection $\Pi^{a}\left(\boldsymbol{v}^{h}\right)$ is the solution of the variational problem

$$
\int_{E} \boldsymbol{\sigma}(\boldsymbol{q}): \boldsymbol{\varepsilon}\left(\Pi^{a} \boldsymbol{v}^{h}\right) d \boldsymbol{x}=\int_{E} \boldsymbol{\sigma}(\boldsymbol{q}): \boldsymbol{\varepsilon}\left(\boldsymbol{v}^{h}\right) d \boldsymbol{x} \quad \forall \boldsymbol{q} \in\left[\mathbb{P}^{1}(E)\right]^{2},
$$

with the additional conditions

$$
\begin{aligned}
& \overline{\Pi^{a} \boldsymbol{v}^{h}}=\overline{\boldsymbol{v}^{h}}, \\
&{\overline{\left(\Pi^{a} \boldsymbol{v}^{h}\right)_{R}}}={\overline{\left(\boldsymbol{v}^{h}\right)_{R}}} .
\end{aligned}
$$

Conditions $111 \mathrm{~b}$ ) and (11c) fix the rigid-body modes (two translations and one rotation) that form the kernel of $\boldsymbol{\varepsilon}(\cdot)$.

A requirement for such a projection operator is that it is computable from the degrees of freedom of $\boldsymbol{V}^{h}$, as we explain below. In order to compute $\Pi^{a}\left(\boldsymbol{v}^{h}\right)$ it is convenient to choose, as a basis of $\mathbb{P}^{1}(E)$, the set of scaled monomials

$$
\boldsymbol{m}(\boldsymbol{x})=\{1, \xi(\boldsymbol{x}), \eta(\boldsymbol{x})\}, \quad \text { with } \quad \xi(\boldsymbol{x})=\frac{x-x_{E}}{h_{E}}, \quad \eta(\boldsymbol{x})=\frac{y-y_{E}}{h_{E}},
$$

where $\boldsymbol{x}=(x, y)^{T}$, so that the basis functions of $\mathbb{P}^{1}(E)$ scale as $O(1)$ with respect to $h$. It immediately follows that $\mathbb{P}^{1}(E)=\operatorname{span}\{1, \xi, \eta\}$, and a possible basis of $\left[\mathbb{P}^{1}(E)\right]^{2}$ is

$$
\left[\mathbb{P}^{1}(E)\right]^{2}=\operatorname{span}\left\{\left(\begin{array}{l}
1 \\
0
\end{array}\right),\left(\begin{array}{c}
0 \\
1
\end{array}\right),\left(\begin{array}{c}
\eta \\
-\xi
\end{array}\right),\left(\begin{array}{l}
\xi \\
0
\end{array}\right),\left(\begin{array}{l}
0 \\
\eta
\end{array}\right),\left(\begin{array}{l}
\eta \\
\xi
\end{array}\right)\right\}
$$

The six vector fields in (13) represent the three planar rigid-body modes and the three independent nonzero deformation modes.

To prove the computability of $\Pi^{a}$, we rewrite $111 \mathrm{a}$ ) with $(11 \mathrm{~b})-(11 \mathrm{c})$ as a linear system. For every $\boldsymbol{\varphi}_{i}$ from the canonical basis of $\boldsymbol{V}^{h}(E)$ shown in (7), we consider the expansion of $\Pi^{a} \boldsymbol{\varphi}_{i}$ on the basis of $\left[\mathbb{P}^{1}(E)\right]^{2}$ shown in (13). A suitable application of the divergence theorem shows that $\Pi^{a} \boldsymbol{\varphi}_{i}$ is computable by using only the degrees of freedom of $\boldsymbol{\varphi}_{i}$ and noting that $\nabla \cdot \sigma\left(\boldsymbol{\varphi}_{i}\right)=\mathbf{0}$. The polynomial projection $\Pi^{a} \boldsymbol{v}^{h}$ can readily be computed for all virtual element fields $\boldsymbol{v}^{h}$ from the projections of the basis functions $\varphi_{i}$ because the projection operator is a linear operator.

We will expand on this observation in the next section. 
Once computed, operator $\Pi^{a}$ allows us to evaluate the local approximated bilinear form as follows

$$
\begin{aligned}
a^{h, E}\left(\boldsymbol{v}^{h}, \boldsymbol{w}^{h}\right) & =a^{E}\left(\Pi^{a}\left(\boldsymbol{v}^{h}\right), \Pi^{a}\left(\boldsymbol{w}^{h}\right)\right)+S^{E}\left(\left(\boldsymbol{v}^{h}-\Pi^{a}\left(\boldsymbol{v}^{h}\right)\right),\left(\boldsymbol{w}^{h}-\Pi^{a}\left(\boldsymbol{w}^{h}\right)\right)\right) \\
& =\int_{E} \boldsymbol{\sigma}\left(\Pi^{a}\left(\boldsymbol{v}^{h}\right)\right): \boldsymbol{\varepsilon}\left(\Pi^{a}\left(\boldsymbol{w}^{h}\right) d \boldsymbol{x}+S^{E}\left(\left(\boldsymbol{v}^{h}-\Pi^{a}\left(\boldsymbol{v}^{h}\right)\right),\left(\boldsymbol{w}^{h}-\Pi^{a}\left(\boldsymbol{w}^{h}\right)\right)\right),\right.
\end{aligned}
$$

where $S^{E}(\cdot, \cdot)$ is a suitable stabilizing term that preserves the coercivity of the system. According to the virtual element methodology, $S^{E}(\cdot, \cdot)$ can be any symmetric, positive definite, continuous bilinear form defined on the kernel of the projection operator $\Pi^{a}[12]$.

We refer the reader to Section 4 for possible choices of the stabilization term.

Finally, the expression for the virtual element approximation of the linear functional in the right-hand side of (8) is given by

$$
b^{h, E}\left(\boldsymbol{v}^{h}\right)=\int_{\Gamma^{t} \cap \partial E} \overline{\boldsymbol{t}} \cdot \boldsymbol{v}^{h} d \Gamma=b^{E}\left(\boldsymbol{v}^{h}\right),
$$

where $b^{h, E}\left(\boldsymbol{v}^{h}\right)$ is computable because $\overline{\boldsymbol{t}}$ is known and the trace of $\boldsymbol{v}^{h}$ is a linear polynomial on each edge $e \in \Gamma^{t} \cap \partial E$ that is known through the interpolation of the edge degrees of freedom.

\subsection{Extended virtual element space, elliptic projection and bilinear form}

If the exact solution to the selected problem contains singularities, then similar to the finite element method, the accuracy of the virtual element method is compromised. For this reason, it is beneficial to enrich the virtual element space by means of independent fields carrying information about the singularities affecting the exact solution. As we discuss later on, such fields are required to satisfy the equilibrium equations (1a). For two-dimensional elastic fracture problems, we choose the enrichment fields as a scaled form of the exact asymptotic crack-tip displacement fields for mode I and mode II crack opening, $\boldsymbol{u}^{I}=\left(u_{x}^{I}, u_{y}^{I}\right)^{T}$ and $\boldsymbol{u}^{I I}=\left(u_{x}^{I I}, u_{y}^{I I}\right)^{T}$, respectively. These enrichment fields are given by the expressions:

$$
\begin{aligned}
& u_{x}^{I}:=u_{x}^{I}(r, \theta)=\sqrt{\frac{r}{2 \pi}}\left[(2 \kappa-1) \cos \left(\frac{\theta}{2}\right)-\cos \left(\frac{3 \theta}{2}\right)\right], \\
& u_{y}^{I}:=u_{y}^{I}(r, \theta)=\sqrt{\frac{r}{2 \pi}}\left[(2 \kappa+1) \sin \left(\frac{\theta}{2}\right)-\sin \left(\frac{3 \theta}{2}\right)\right], \\
& u_{x}^{I I}:=u_{x}^{I I}(r, \theta)=\sqrt{\frac{r}{2 \pi}}\left[(2 \kappa+3) \sin \left(\frac{\theta}{2}\right)+\sin \left(\frac{3 \theta}{2}\right)\right], \\
& u_{y}^{I I}:=u_{y}^{I I}(r, \theta)=-\sqrt{\frac{r}{2 \pi}}\left[(2 \kappa-3) \cos \left(\frac{\theta}{2}\right)+\cos \left(\frac{3 \theta}{2}\right)\right],
\end{aligned}
$$

where $(r, \theta)$ are polar coordinates in the local crack tip reference system (see Fig. 2) and $\kappa$ is the Kolosov constant.

An explicit computation implies that these fields satisfy equilibrium, i.e., the conditions $\nabla \cdot \sigma\left(\boldsymbol{u}^{I}\right)=0$ and $\nabla \cdot \sigma\left(\boldsymbol{u}^{I I}\right)=0$ hold. Note that $\boldsymbol{u}^{I}$ and $\boldsymbol{u}^{I I}$ belong to $H^{\frac{3}{2}-\eta}(\Omega)$ for any $\eta>0[38]$, 


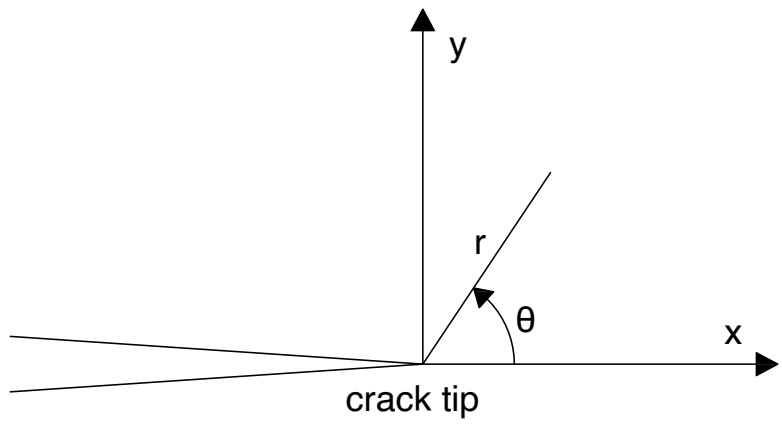

Figure 2: Local crack-tip reference system in polar coordinates.

and this fact reduces the convergence rate of a standard finite element or virtual element method to $O\left(h^{\frac{1}{2}}\right)$.

Let $\check{\boldsymbol{u}}^{I}=\left(\check{u}_{x}^{I}, \check{u}_{y}^{I}\right)^{T}$ and $\check{\boldsymbol{u}}^{I I}=\left(\check{u}_{x}^{I I}, \check{u}_{y}^{I I}\right)^{T}$ denote the dimensionless version of fields $\boldsymbol{u}^{I}$ and $\boldsymbol{u}^{I I}$, respectively,

$$
\check{\boldsymbol{u}}^{I}=\boldsymbol{u}^{I} / h^{1 / 2} \text { and } \check{\boldsymbol{u}}^{I I}=\boldsymbol{u}^{I I} / h^{1 / 2},
$$

where $h=\max _{E \in \Omega_{h}}\left(h_{E}\right)$. In order to define the extended virtual element space, we first introduce the local virtual element space $\boldsymbol{V}^{h, *}(E) \subset \boldsymbol{V}^{h}(E)$, which reads as

$$
\boldsymbol{V}^{h, *}(E) \equiv\left\{\boldsymbol{v}^{h}=\left(v_{x}^{h}, v_{y}^{h}\right)^{T} \in \boldsymbol{V}^{h}(E): v_{x}^{h}=v_{y}^{h}\right\} .
$$

This space is generated by the linear combination of the basis functions $\varphi_{i}^{*}=\left(\varphi_{i}, \varphi_{i}\right)^{T}, i=$ $1, \ldots, N_{E}$, where the functions $\varphi_{i}$ are the basis functions of the scalar virtual element space $V^{h}(E)$, so that $\boldsymbol{V}^{h, *}(E)=\operatorname{span}\left\{\boldsymbol{\varphi}_{1}^{*}, \ldots, \boldsymbol{\varphi}_{N_{E}}^{*}\right\}$. The dimension of this space is clearly $N_{E}$ and the partition-of-unity property of functions $\varphi_{i}$ implies that

$$
\sum_{i=1}^{N_{E}} \boldsymbol{\varphi}_{i}^{*}(\boldsymbol{x})=\left(\begin{array}{c}
\sum_{i=1}^{N_{E}} \phi_{i}(\boldsymbol{x}) \\
\sum_{i=1}^{N_{E}} \phi_{i}(\boldsymbol{x})
\end{array}\right)=\left(\begin{array}{c}
1 \\
1
\end{array}\right) \quad \forall \boldsymbol{x} \in E .
$$

Then, we define the matrices $\psi^{I}$ and $\psi^{I I}$ as

$$
\boldsymbol{\psi}^{I} \equiv\left[\begin{array}{cc}
\check{u}_{x}^{I} & 0 \\
0 & \check{u}_{y}^{I}
\end{array}\right], \quad \boldsymbol{\psi}^{I I} \equiv\left[\begin{array}{cc}
\check{u}_{x}^{I I} & 0 \\
0 & \check{u}_{y}^{I I}
\end{array}\right] .
$$

We now have all the ingredients to define the local extended virtual element space $V_{X}^{h}(E)$, which reads as

$$
\boldsymbol{V}_{X}^{h}(E) \equiv \boldsymbol{V}^{h}(E) \oplus \boldsymbol{\psi}^{I} \boldsymbol{V}^{h, *}(E) \oplus \boldsymbol{\psi}^{I I} \boldsymbol{V}^{h, *}(E) .
$$

We obtain a basis of this space as the union of the basis functions of $\boldsymbol{V}_{X}^{h}(E), \boldsymbol{\psi}^{I} \boldsymbol{V}^{h, *}(E)$ and $\boldsymbol{\psi}^{I I} \boldsymbol{V}^{h, *}(E)$, so that

$$
\boldsymbol{V}_{X}^{h}(E)=\operatorname{span}\left\{\boldsymbol{\varphi}_{1}, \boldsymbol{\varphi}_{2}, \ldots, \boldsymbol{\varphi}_{2 i-1}, \boldsymbol{\varphi}_{2 i}, \ldots, \boldsymbol{\varphi}_{2 N_{E}-1}, \boldsymbol{\varphi}_{2 N_{E}}\right\} \cup \boldsymbol{\psi}^{I} \operatorname{span}\left\{\boldsymbol{\varphi}_{1}^{*}, \boldsymbol{\varphi}_{2}^{*}, \ldots, \boldsymbol{\varphi}_{N_{E}}^{*}\right\}
$$




$$
\cup \psi^{I I} \operatorname{span}\left\{\varphi_{1}^{*}, \varphi_{2}^{*}, \ldots, \varphi_{N_{E}}^{*}\right\},
$$

where we recall that $\varphi_{2 i-1}=\left(\varphi_{i}, 0\right)^{T}, \varphi_{2 i}=\left(0, \varphi_{i}\right)^{T}$ and $\varphi_{i}^{*}=\left(\varphi_{i}, \varphi_{i}\right)^{T}, i=1, \ldots, N_{E}$. Therefore, at every enriched node the vector-valued field $\boldsymbol{v}_{X}^{h}(\boldsymbol{x})$ that belongs to the extended virtual element space $\boldsymbol{V}_{X}^{h}(E)$ is characterized by four values and for an element whose nodes are all enriched, we have $4 N_{E}$ degrees of freedom. For example, at the $j$-th node with coordinates $\boldsymbol{x}_{j}$, we find that

$$
\begin{aligned}
\boldsymbol{v}^{h}\left(\boldsymbol{x}_{j}\right) & =\sum_{i=1}^{N_{E}}\left[v_{i, x}^{h}\left(\begin{array}{c}
\varphi_{i}\left(\boldsymbol{x}_{j}\right) \\
0
\end{array}\right)+v_{i, y}^{h}\left(\begin{array}{c}
0 \\
\varphi_{i}\left(\boldsymbol{x}_{j}\right)
\end{array}\right)+v_{i, I}^{h}\left(\begin{array}{c}
\check{u}_{x}^{I}\left(\boldsymbol{x}_{j}\right) \varphi_{i}\left(\boldsymbol{x}_{j}\right) \\
\check{u}_{y}^{I}\left(\boldsymbol{x}_{j}\right) \varphi_{i}\left(\boldsymbol{x}_{j}\right)
\end{array}\right)+v_{i, I I}^{h}\left(\begin{array}{c}
\check{u}_{x}^{I I}\left(\boldsymbol{x}_{j}\right) \varphi_{i}\left(\boldsymbol{x}_{j}\right) \\
\check{u}_{y}^{I I}\left(\boldsymbol{x}_{j}\right) \varphi_{i}\left(\boldsymbol{x}_{j}\right)
\end{array}\right)\right] \\
& =\left(\begin{array}{c}
v_{j, x}^{h}+v_{j, I}^{h} \check{u}_{x}^{I}\left(\boldsymbol{x}_{j}\right)+v_{j, h}^{h} \check{u}_{x}^{I I}\left(\boldsymbol{x}_{j}\right) \\
v_{j, y}^{h}+v_{j, I I}^{h} \check{u}_{y}^{I}\left(\boldsymbol{x}_{j}\right)+v_{j, I I}^{h} \check{u}_{y}^{I I}\left(\boldsymbol{x}_{j}\right)
\end{array}\right),
\end{aligned}
$$

since $\varphi_{i}\left(\boldsymbol{x}_{j}\right)=\delta_{i j}$.

Remark 3.1. Here, $v_{i, x}^{h}, v_{i, y}^{h}, v_{i, I}^{h}$ and $v_{i, I I}^{h}$ are the coefficients of the basis functions in (19) and can thus be identified with the degrees of freedom of the method. Note, however, that the degrees of freedom of an enriched function $\boldsymbol{v}_{X}^{h} \in \boldsymbol{V}_{X}^{h}(E)$ are no longer the values of $\boldsymbol{v}_{X}^{h}$ at the vertices of element $E$.

To ease the exposition, we denote the basis functions of $\boldsymbol{V}_{X}^{h}(E)$ by the symbol $\boldsymbol{\varphi}_{i}, i=$ $1,2, \ldots, 4 N_{E}$, so that $\boldsymbol{V}_{X}^{h}(E)=\operatorname{span}\left\{\boldsymbol{\varphi}_{1}, \boldsymbol{\varphi}_{2}, \ldots, \boldsymbol{\varphi}_{4 N_{E}}\right\}$ where

$$
\boldsymbol{\varphi}_{i}= \begin{cases}\left(\varphi_{i}, 0\right)^{T} & \text { for } 1 \leq i \leq 2 N_{E}, i \text { odd } \\ \left(0, \varphi_{i}\right)^{T} & \text { for } 1 \leq i \leq 2 N_{E}, i \text { even } \\ \left(\check{u}_{x}^{I} \varphi_{i}, \check{u}_{y}^{I} \varphi_{i}\right)^{T} & \text { for } 1+2 N_{E} \leq i \leq 3 N_{E}, \\ \left(\check{u}_{x}^{I I} \varphi_{i}, \check{u}_{y}^{I I} \varphi_{i}\right)^{T} & \text { for } 1+3 N_{E} \leq i \leq 4 N_{E} .\end{cases}
$$

Finally, the extended global virtual element space $\boldsymbol{V}_{X}^{h}$ is defined as follows:

$$
\boldsymbol{V}_{X}^{h}=\left\{\boldsymbol{v}_{X}^{h} \in\left[H^{1}(\Omega)\right]^{2}: \boldsymbol{v}_{X \mid E}^{h} \in \boldsymbol{V}_{X}^{h}(E) \quad \forall E \in \Omega_{h}\right\} .
$$

Again, to consider the essential boundary condition (1d) we consider the affine subspace $V_{X, g}^{h}$ of $\boldsymbol{V}_{X}^{h}$ defined by

$$
\boldsymbol{V}_{X, \boldsymbol{g}}^{h}=\left\{\boldsymbol{v}_{X}^{h} \in \boldsymbol{V}_{X}^{h}: \boldsymbol{v}_{X}^{h}=\boldsymbol{g}_{X}^{h} \text { on } \Gamma_{u}\right\},
$$

where $\boldsymbol{g}_{X}^{h}$ is the extended linear interpolant of $\boldsymbol{g}$, and the linear subspace $\boldsymbol{V}_{X, \mathbf{0}}^{h}$, which is defined by setting $\boldsymbol{g}_{X}^{h}=\mathbf{0}$ in the above definition.

Since $\left\{\boldsymbol{\varphi}_{i}\right\}_{i=1}^{4 N_{E}}$ are not known in the interior of the element, we construct a convenient projection operator that will allow us to obtain computable approximations $a_{X}^{h}(\cdot, \cdot): V_{X}^{h}(E) \times V_{X}^{h}(E) \rightarrow$ $\mathbb{R}$ and $b_{X}^{h}(\cdot): V_{X}^{h}(E) \rightarrow \mathbb{R}$ of the exact bilinear form $a(\cdot, \cdot)$ and the linear functional $b(\cdot)$ appearing in (4). The extended virtual element formulation then reads: Find $\boldsymbol{u}_{X}^{h} \in \boldsymbol{V}_{X, g}^{h}$ such that

$$
a_{X}^{h}\left(\boldsymbol{u}_{X}^{h}, \boldsymbol{v}_{X}^{h}\right)=b_{X}^{h}\left(\boldsymbol{v}_{X}^{h}\right) \quad \forall \boldsymbol{v}_{X}^{h} \in \boldsymbol{V}_{X, 0}^{h},
$$


where the bilinear form $a_{X}^{h}(\cdot, \cdot)$ is built element-wise as

$$
a_{X}^{h}\left(\boldsymbol{u}_{X}^{h}, v_{X}^{h}\right)=\sum_{E \in \Omega} a_{X}^{h, E}\left(\boldsymbol{u}_{X}^{h}, \boldsymbol{v}_{X}^{h}\right) \quad \forall \boldsymbol{u}_{X}^{h}, \boldsymbol{v}_{X}^{h} \in \boldsymbol{V}_{X}^{h}
$$

and again we set $b_{X}^{h}\left(v_{X}^{h}\right)=b\left(v_{X}^{h}\right)$.

In order to construct a consistent and stable bilinear form $a_{X}^{h, E}(\cdot, \cdot)$, we extend the polynomial space $\mathbb{P}^{1}(E)$ to a subspace of $V^{h}(E)$ including the linear polynomials and the additional enrichment functions $\check{\boldsymbol{u}}^{I}$ and $\check{\boldsymbol{u}}^{I I}$, so that

$$
\mathbb{P}_{X}(E) \equiv \mathbb{P}^{1}(E) \oplus \operatorname{span}\left(\check{\boldsymbol{u}}^{I}, \check{\boldsymbol{u}}^{I I}\right) .
$$

Space $\mathbb{P}_{X}(E)$ is spanned by the eight linearly independent vector fields

$$
\mathbb{P}_{X}(E)=\operatorname{span}\left\{\left(\begin{array}{l}
1 \\
0
\end{array}\right),\left(\begin{array}{c}
0 \\
1
\end{array}\right),\left(\begin{array}{c}
\eta \\
-\xi
\end{array}\right),\left(\begin{array}{c}
\xi \\
0
\end{array}\right),\left(\begin{array}{l}
0 \\
\eta
\end{array}\right),\left(\begin{array}{c}
\eta \\
\xi
\end{array}\right),\left(\begin{array}{c}
\check{u}_{x}^{I} \\
\check{u}_{y}^{I}
\end{array}\right),\left(\begin{array}{c}
\check{u}_{x}^{I I} \\
\check{u}_{y}^{I I}
\end{array}\right)\right\} .
$$

The first six vector fields in (22) represent the three fundamental rigid body motions and the three independent deformation modes that form $\mathbb{P}^{1}(E)$, cf. (13). The last two vector fields are the scaled enrichment fields chosen to construct the extended virtual element space $V_{X}^{h}(E)$.

Remark 3.2. All $\boldsymbol{q}_{X} \in \mathbb{P}_{X}(E)$ satisfy the equilibrium equation $\nabla \cdot \boldsymbol{\sigma}\left(\boldsymbol{q}_{X}\right)=\mathbf{0}$. This property is crucial to determine the computability of the extended projection operator $\Pi_{X}^{a}$.

To construct a bilinear form $a_{X}^{h, E}(\cdot, \cdot)$ for which such properties hold, we define the extended elliptic projection operator $\Pi_{X}^{a}: \boldsymbol{V}_{X}^{h}(E) \rightarrow \mathbb{P}_{X}(E)$ for each element $E$. For each $\boldsymbol{v}_{X}^{h} \in \boldsymbol{V}_{X}^{h}(E)$, the extended elliptic projection $\Pi_{X}^{a}\left(v_{X}^{h}\right)$ is the solution of the variational problem

$$
\int_{E} \boldsymbol{\sigma}\left(\boldsymbol{q}_{X}\right): \boldsymbol{\varepsilon}\left(\Pi_{X}^{a} \boldsymbol{v}_{X}^{h}\right) d \boldsymbol{x}=\int_{E} \boldsymbol{\sigma}\left(\boldsymbol{q}_{X}\right): \boldsymbol{\varepsilon}\left(\boldsymbol{v}_{X}^{h}\right) d \boldsymbol{x} \quad \forall \boldsymbol{q}_{X} \in \mathbb{P}_{X}(E),
$$

with the additional conditions

$$
\begin{aligned}
\overline{\Pi_{X}^{a} v_{X}^{h}} & =\overline{v_{X}^{h}}, \\
\overline{\left(\Pi_{X}^{a} v_{X}^{h}\right)_{R}} & =\overline{\left(v_{X}^{h}\right)_{R}},
\end{aligned}
$$

where $\overline{(\cdot)}$ and $\overline{(\cdot)}_{R}$ are the average translation and rotation, respectively, which are defined in (9) and (10). Recalling the divergence theorem and Remark 3.2, the vector polynomial $\Pi_{X}^{a} v_{X}^{h} \in$ $\mathbb{P}_{X}(E)$ is computable from the degrees of freedom of $v_{X}^{h}$.

The projection operator $\Pi_{X}^{a}$ allows us to define the local extended bilinear form as follows:

$$
\begin{aligned}
a_{X}^{h, E}\left(\boldsymbol{v}_{X}^{h}, \boldsymbol{w}_{X}^{h}\right) & \equiv a^{E}\left(\Pi_{X}^{a}\left(\boldsymbol{v}_{X}^{h}\right), \Pi_{X}^{a}\left(\boldsymbol{w}_{X}^{h}\right)\right)+S_{X}^{E}\left(\boldsymbol{v}_{X}^{h}-\Pi_{X}^{a}\left(\boldsymbol{v}_{X}^{h}\right), \boldsymbol{w}_{X}^{h}-\Pi_{X}^{a}\left(\boldsymbol{w}_{X}^{h}\right)\right) \\
& =\int_{E} \boldsymbol{\sigma}\left(\Pi_{X}^{a}\left(\boldsymbol{v}_{X}^{h}\right)\right): \boldsymbol{\varepsilon}\left(\Pi_{X}^{a}\left(\boldsymbol{w}_{X}^{h}\right)\right) d \boldsymbol{x}+S_{X}^{E}\left(\boldsymbol{v}_{X}^{h}-\Pi_{X}^{a}\left(\boldsymbol{v}_{X}^{h}\right), \boldsymbol{w}_{X}^{h}-\Pi_{X}^{a}\left(\boldsymbol{w}_{X}^{h}\right)\right),
\end{aligned}
$$

where $S_{X}^{E}(\cdot, \cdot)$ is a stabilization term that must be suitably defined to guarantee linear consistency (cf. (25) ) and stability (cf. (26) ) of the method. Again, according to the virtual element 
methodology, $S_{X}^{E}(\cdot, \cdot)$ can be any symmetric, positive definite, continuous bilinear form defined on the kernel of the extended projection operator $\Pi_{X}^{a}[15]$. The reader is referred to Section 4 for possible choices of the stabilization term.

With a suitable choice of the stabilization term, the bilinear form $a_{X}^{h, E}(\cdot, \cdot)$ has the following properties, which are fundamental in order to guarantee the convergence of the method:

(i) extended linear consistency: for all $\boldsymbol{v}_{X}^{h} \in \boldsymbol{V}_{X}^{h}(E)$ and $\boldsymbol{q}_{X} \in \mathbb{P}_{X}(E)$ it holds that

$$
a_{X}^{h, E}\left(\boldsymbol{v}_{X}^{h}, \boldsymbol{q}_{X}\right)=a^{E}\left(\boldsymbol{v}_{X}^{h}, \boldsymbol{q}_{X}\right)
$$

(ii) stability: there exist two positive constants $\alpha_{*}, \alpha^{*}$, independent of $h$ and $E$, such that

$$
\alpha_{*} a^{E}\left(\boldsymbol{v}_{X}^{h}, \boldsymbol{v}_{X}^{h}\right) \leq a_{X}^{h, E}\left(\boldsymbol{v}_{X}^{h}, \boldsymbol{v}_{X}^{h}\right) \leq \alpha^{*} a^{E}\left(\boldsymbol{v}_{X}^{h}, \boldsymbol{v}_{X}^{h}\right) \quad \forall \boldsymbol{v}_{X}^{h} \in \boldsymbol{V}_{X}^{h}(E) .
$$

According to the virtual element theory, cf. [12], the constants $\alpha_{*}$ and $\alpha^{*}$ must be independent of the mesh size parameter $h$. However, they can depend on the other model and discretization parameters such as the bound on $\boldsymbol{C}$ and the mesh regularity constant $\rho$. Here, $a^{E}(\cdot, \cdot)$ is the local coercive and continuous bilinear form

$$
a^{E}(\boldsymbol{u}, \boldsymbol{v})=\int_{E} \boldsymbol{\sigma}(\boldsymbol{v}): \boldsymbol{\varepsilon}(\boldsymbol{u}) d \boldsymbol{x} \quad \forall \boldsymbol{u}, \boldsymbol{v} \in \mathscr{U}_{0}
$$

Remark 3.3. In Section 4 we provide two possible choices of the stabilization term by considering the standard dofi-dofi and D-recipe formulations in our extended setting. Such choices are widely accepted in the VEM literature and in some cases they were theoretically proved to be effective to guarantee stability relations such as (26). However, the choice of the stabilization term in the presence of enrichment functions and its impact on the behavior of the VEM are still open issues at this time. For example, it would be desirable that the constants of the stability relation (26) are independent of the Young's modulus and Poisson's ratio to realize a robust discretization. These topics will be the subject of future work.

\subsection{Partial enrichment}

Let $E$ denote an element of mesh $\Omega_{h}$ and $k_{E}$ a positive integer number strictly less than $N_{E}$ (the case for $k_{E}=N_{E}$ is the full enrichment case). We select $k_{E}$ distinct nodes of element $E$ to be enriched and the corresponding basis functions $\varphi_{i_{\ell}}^{*} \in V^{h, *}(E)$ labeled by the $k_{E}$ distinct indices $i_{\ell} \in\left[1, N_{E}\right]$ for $\ell=1, \ldots, k_{E}$. We formally denote the subset of these indices by $\mathcal{I}=\left\{i_{\ell}\right\}_{\ell=1}^{k_{E}}$. Using these basis functions, we define the reduced virtual element space

$$
\widetilde{\boldsymbol{V}}^{h, *}(E) \equiv \operatorname{span}\left\{\boldsymbol{\varphi}_{i_{1}}^{*}, \boldsymbol{\varphi}_{i_{2}}^{*}, \ldots, \boldsymbol{\varphi}_{i_{k_{E}}}^{*}\right\} \subset \boldsymbol{V}^{h, *}(E)
$$

and the reduced extended virtual element space

$$
\widetilde{\boldsymbol{V}}_{X}^{h}(E)=\boldsymbol{V}^{h}(E) \oplus \boldsymbol{\psi}^{I} \widetilde{\boldsymbol{V}}^{h, *}(E) \oplus \boldsymbol{\psi}^{I I} \widetilde{\boldsymbol{V}}^{h, *}(E) \subset \boldsymbol{V}_{X}^{h},
$$

where a tilde accent as a superscript is used to denote all 'reduced' mathematical objects. Equivalently, we can define the reduced virtual element space $\widetilde{\boldsymbol{V}}_{X}^{h}(E)$ as the span of the basis functions of $\boldsymbol{V}^{h}(E), \psi^{I} \widetilde{\boldsymbol{V}}^{h, *}(E)$ and $\psi^{I I} \widetilde{\boldsymbol{V}}^{h, *}(E)$, so that

$$
\widetilde{\boldsymbol{V}}_{X}^{h}(E)=\operatorname{span}\left\{\boldsymbol{\varphi}_{1}, \boldsymbol{\varphi}_{2}, \ldots, \boldsymbol{\varphi}_{2 i-1}, \boldsymbol{\varphi}_{2 i}, \ldots, \boldsymbol{\varphi}_{2 N_{E}-1}, \boldsymbol{\varphi}_{2 N_{E}}\right\} \cup \boldsymbol{\psi}^{I} \operatorname{span}\left\{\boldsymbol{\varphi}_{1}^{*}, \boldsymbol{\varphi}_{2}^{*}, \ldots, \boldsymbol{\varphi}_{k_{E}}^{*}\right\}
$$




$$
\cup \psi^{I I} \operatorname{span}\left\{\varphi_{1}^{*}, \varphi_{2}^{*}, \ldots, \varphi_{k_{E}}^{*}\right\}
$$

which can be compared to (19). Accordingly, a generic virtual element function that belongs to the reduced space $\widetilde{\boldsymbol{V}}_{X}^{h}(E)$ is described by $2 N_{E}+2 k_{E}$ degrees of freedom instead of $4 N_{E}$ degrees of freedom. The first $2 N_{E}$ degrees of freedom are the vertex values of a vector-valued field $v^{h} \in V^{h}(E)$. The other $2 k_{E}$ degrees of freedom correspond to the vertex values of a virtual vectorvalued function that belongs to the enriching space $\psi^{I} \widetilde{\boldsymbol{V}}^{h, *}(E) \oplus \boldsymbol{\psi}^{I I} \widetilde{\boldsymbol{V}}^{h, *}(E)$ and clearly depends on $\check{\boldsymbol{u}}^{I}$ and $\check{\boldsymbol{u}}^{I I}$. We outline a few important facts that will be crucial in the implementation of the partially enriched virtual element method. First, the set of basis functions $\varphi_{i_{\ell}}^{*}$ for $\ell=$ $1, \ldots, k_{E}$ does not satisfy a partition-of-unity property. Consequently, the enriching fields $\check{\boldsymbol{u}}^{I}$ and $\check{\boldsymbol{u}}^{I I}$ are not elements of $\boldsymbol{\psi}^{I} \widetilde{\boldsymbol{V}}^{h, *}(E) \oplus \boldsymbol{\psi}^{I I} \widetilde{\boldsymbol{V}}^{h, *}(E)$ and the extended space $\mathbb{P}_{X}(E)$ cannot be a subspace of $\widetilde{\boldsymbol{V}}^{h, *}(E)$. However, since $\widetilde{\boldsymbol{V}}_{X}^{h}(E)$ is a linear subspace of $\boldsymbol{V}_{X}^{h}(E)$, we can still apply the projection operator $\Pi_{X}^{a}$ to its functions and obtain a projection in the extended space $\mathbb{P}_{X}(E)$, and the construction of the bilinear form $a_{X}^{h, E}(\cdot, \cdot)$ of the previous section still holds. For a proper formal definition, we introduce the extension (or injection) operator $\mathcal{E}_{k_{E}}: \widetilde{\boldsymbol{V}}^{h, *}(E) \rightarrow \boldsymbol{V}^{h, *}(E)$ that remaps any reduced virtual element function $\widetilde{\boldsymbol{v}}_{X}^{h} \in \widetilde{\boldsymbol{V}}_{X}^{h}(E)$ into the fully enriched function $\left.\mathcal{E}_{k_{E}} \widetilde{\boldsymbol{v}}_{X}^{h}\right) \in \boldsymbol{V}_{X}^{h}(E)$ such that:

$$
i \text {-th DOF of } \mathcal{E}_{k_{E}}\left(\overrightarrow{\boldsymbol{v}}_{X}^{h}\right)= \begin{cases}i \text {-th DOF of } \widetilde{\boldsymbol{v}}_{X}^{h} & \text { if } 1 \leq i \leq 2 N_{E}, \\ i_{\ell} \text {-th DOF of } \widetilde{\boldsymbol{v}}_{X}^{h} & \text { if } i=2 N_{E}+i_{\ell} \text { or } i=3 N_{E}+i_{\ell} \text { with } i_{\ell} \in \mathcal{I}, \\ 0 & \text { otherwise. }\end{cases}
$$

Practically speaking, the remapped function has the same degrees of freedom of the reduced functions and zero at all the additional degrees of freedom that correspond to the nonenriched nodes. Then, we define a new stiffness bilinear form $\widetilde{a}_{X}^{h, E}(\cdot, \cdot): \widetilde{\boldsymbol{V}}_{X}^{h}(E) \times \widetilde{\boldsymbol{V}}_{X}^{h}(E) \rightarrow \mathbb{R}$ as

$$
\left.\widetilde{a}_{X}^{h, E}\left(\widetilde{\boldsymbol{v}}_{X}^{h}, \widetilde{\boldsymbol{w}}_{X}^{h}\right):=a_{X}^{h, E}\left(\mathcal{E}_{k_{E}} \widetilde{\boldsymbol{v}}_{X}^{h}\right), \mathcal{E}_{k_{E}}\left(\widetilde{\boldsymbol{w}}_{X}^{h}\right)\right),
$$

so that we can reuse the definition of $a_{X}^{h, E}(\cdot, \cdot)$. Furthermore, the whole construction of the previous section, including the consistency and stability properties, still holds.

As we discuss in the implementation section, this formal approach also suggests a straightforward way (but perhaps not the most efficient one) to implement the partial enrichment as all we need in practice is to apply a matrix representation of the injection operator $\mathcal{E}_{k_{E}}$ to the element stiffness matrix of a fully enriched element. We will see that this procedure is equivalent to first constructing the fully enriched stiffness matrix, and then simply suppressing all rows and columns that correspond to the degrees of freedom of the nonenriched nodes.

As we note in Section 5, partial enrichment induces a loss of optimal convergence, which also occurs in the X-FEM. This consequence is not surprising, since even though we are projecting onto a space consisting of polynomials and nonpolynomial near-tip enrichment fields, in this case the local extended virtual element space is not sufficiently rich to approximate the singular behaviour of the function near the crack tip. Special enrichment strategies can be devised to overcome this issue, for instance using the so-called geometric enrichment. 


\subsection{Embedding discontinuities}

In this section, we show how both the regular and the extended virtual element formulations presented in Sections 3.2 and 3.3 can be endowed with a structure that allows discontinuous fields to be embedded within the virtual element space. Consider a crack $\gamma$ that intersects some of the elements in a mesh, and define $d(\boldsymbol{x})$ as the signed distance from a point $\boldsymbol{x}$ to $\gamma$. For modeling strong discontinuities like a crack, it would be convenient to consider enrichment with the generalized Heaviside function $H(\boldsymbol{x})$, which is equal to +1 for points with $d(\boldsymbol{x}) \geq 0(\boldsymbol{x}$ is on or above the crack) and -1 for points with $d(\boldsymbol{x})<0$ ( $\boldsymbol{x}$ is below the crack). As in the XFEM, we could enrich those nodes whose basis function's support intersects the interior of the crack (not including the tips) with $H(x)$. However, the resulting extended projection $\Pi_{X}^{a}$ onto $\mathbb{P}_{X}(E)$ would not be directly computable from the degrees of freedom of the method because the corresponding enriched virtual element basis functions $H \varphi_{i}$ are not known along the crack.

To deliver a viable solution, we let the element $E$ to be partitioned by the discontinuity $\gamma$ into two subdomains $E^{-}$and $E^{+}$. Following [33], in order to represent two independent linear polynomials on $E^{-}$and $E^{+}$, we adopt the approach of Hansbo and Hansbo [37] and tailor it to the X-VEM. It is known that the approach of Hansbo and Hansbo is equivalent to the standard $\mathrm{X}$-FEM approximation with Heaviside enrichment [39]. To this end, let $N_{\text {dofs }}^{\mathrm{VE}}$ denote the number of degrees of freedom for element $E$, such that $N_{\text {dofs }}^{\mathrm{VE}}=2 N_{E}$ for the virtual element formulation in 3.2 and $N_{\mathrm{dofs}}^{\mathrm{VE}}=4 N_{E}$ for the extended virtual element formulation in Section 3.3. Each one of the $N_{\text {dofs }}^{\mathrm{VE}}$ virtual shape functions, $\varphi_{i}$ on $E$, is written as the sum of two new virtual shape functions $\varphi_{i}^{-}$and $\varphi_{i}^{+}$that are both discontinuous across the crack, and are defined as follows:

$$
\boldsymbol{\varphi}_{i}^{+}=\left\{\begin{array}{ll}
0 & \text { in } E^{-} \\
\varphi_{i} & \text { in } E^{+}
\end{array}, \quad \varphi_{i}^{-}=\left\{\begin{array}{ll}
\varphi_{i} & \text { in } E^{-} \\
0 & \text { in } E^{+}
\end{array} .\right.\right.
$$

Clearly, $\varphi_{i}^{-}$and $\varphi_{i}^{+}$are harmonic and continuous functions in $E^{-}$and $E^{+}$, respectively, and $\boldsymbol{\varphi}_{i}=\boldsymbol{\varphi}_{i}^{-}+\boldsymbol{\varphi}_{i}^{+}$. Proceeding likewise for all the degrees of freedom in the element, we can generate $N_{\text {dofs }}^{\mathrm{HH}}=2 N_{\text {dofs }}^{\mathrm{VE}}$ discontinuous functions, starting from the initial $N_{\text {dofs }}^{\mathrm{VE}}$ virtual basis functions. This choice implies doubling the nodal DOFs of the element. Therefore, the number of degrees of freedom for the element with an internal discontinuity is twice that of the original element, and a virtual element basis is constructed by considering two copies of the original virtual element basis functions, restricted to $E^{-}$and $E^{+}$respectively, as defined in (30).

We now define the local virtual element space to which the discontinuous approximate solution belongs. For the sake of simplicity, we present the derivation with respect to the formulation presented in Section 3.2. Consider the following spaces:

$$
\begin{gathered}
\boldsymbol{V}^{h,-}(E) \equiv\left\{\boldsymbol{v}^{h} \in\left[H^{1}(E-)\right]^{2}: \Delta \boldsymbol{v}_{\mid E^{-}}=\mathbf{0}, \boldsymbol{v}^{h}{ }_{\mid \partial E^{-}} \in\left[C^{0}\left(\partial E^{-}\right)\right]^{2},\right. \\
\left.\boldsymbol{v}_{\mid e}^{h} \in\left[\mathbb{P}^{1}(e)\right]^{2} \forall e \in\left(\partial E \cap \partial E^{-}\right), \boldsymbol{v}^{h}{ }_{\mid E^{+}}=\mathbf{0}\right\}, \\
\boldsymbol{V}^{h,+}(E) \equiv\left\{\boldsymbol{v}^{h} \in\left[H^{1}\left(E^{+}\right)\right]^{2}: \Delta \boldsymbol{v}_{\mid E^{+}}{ }^{h}=\mathbf{0}, \boldsymbol{v}_{\mid \partial E^{+}} \in\left[C^{0}\left(\partial E^{+}\right)\right]^{2},\right. \\
\left.\boldsymbol{v}_{\mid e} \in\left[\mathbb{P}^{1}(e)\right]^{2} \forall e \in\left(\partial E \cap \partial E^{+}\right), \boldsymbol{v}_{\mid E^{-}}=\mathbf{0}\right\} .
\end{gathered}
$$

Then, the local virtual element space reads:

$$
\boldsymbol{V}_{X}^{h}(E) \equiv\left\{\boldsymbol{v}_{X}^{h}=\left(\boldsymbol{v}^{h,-}+\boldsymbol{v}^{h,+}\right): \boldsymbol{v}^{h,-} \in \boldsymbol{V}^{h,-}(E), \boldsymbol{v}^{h,+} \in \boldsymbol{V}^{h,+}(E)\right\} .
$$


Remark 3.4. The space $\boldsymbol{V}_{X}^{h}(E)$ in (31) is not a subspace of $H^{1}(E)$ as we do not assume any regularity of the virtual element functions across the crack, so that a discontinuity is admissible. This fact implies that also the global virtual element space $V_{X}^{h}$ cannot be a subspace of $H^{1}(\Omega)$, but this is not an issue since the exact solution contains a discontinuity and thus cannot be in $H^{1}(\Omega)$.

An analogous definition of the local virtual element space for elements cut by a crack can be easily provided also for the enriched formulation presented in Section 3.3 .

As we detail later on, virtual element functions along interface edges can be reconstructed by a suitable approximation. We obtain the following representation for the virtual element approximation on the element $E$ cut by $\gamma$ :

$$
\boldsymbol{v}_{X}^{h}(\boldsymbol{x})=\sum_{i=1}^{N_{\mathrm{dof}}^{\mathrm{VE}}}\left[\boldsymbol{\varphi}_{i}^{-}(\boldsymbol{x}) v_{i}^{-}+\boldsymbol{\varphi}_{i}^{+}(\boldsymbol{x}) v_{i}^{+}\right] \quad \forall \boldsymbol{x} \in E,
$$

where $v_{i}^{-}$and $v_{i}^{+}$are the degrees of freedom associated with $\varphi_{i}^{-}$and $\varphi_{i}^{+}$, respectively. To provide a feasible solution using (32), it is necessary to know the trace of the virtual shape functions $\varphi_{i}$ along the crack. We also need two distinct regular projectors, respectively $\Pi^{a,-}$ onto $\left[\mathbb{P}^{1}\left(E^{-}\right)\right]^{2}$ and $\Pi^{a,+}$ onto $\left[\mathbb{P}^{1}\left(E^{+}\right)\right]^{2}$, and two distinct extended projectors, respectively $\Pi_{X}^{a,-}$ onto $\mathbb{P}_{X}\left(E^{-}\right)$ and $\Pi_{X}^{a,+}$ onto $\mathbb{P}_{X}\left(E^{+}\right)$. These projection operators must be computable from the $N_{\text {dof }}^{\mathrm{HH}}$ nodal degrees of freedom. A convenient approximation of the trace of the $i$-th virtual element shape function $\varphi_{i}$ along the crack is provided by a vector-valued function $N_{i}(x)$, that is componentwise harmonic on the cracked element $E$. Such a function is built as a first-order polyharmonic spline [40] and the reader is pointed to [33] for further details.

We also point out that the flexibility of the virtual element method allows an element to be cut into two polygonal virtual elements, regardless of the element shape, and therefore the modeling of crack opening and growth can follow this alternative route (see [29]). However, mesh quality can be affected. For instance, let us consider the case when partitioning of the element results in one subelement being a quasi-degenerate triangle: this badly-shaped triangle will worsen matrix-conditioning and/or the interpolation error. This scenario becomes acute in $3 \mathrm{D}$ if sliver tetrahedra appear and the partitioning is now much more difficult to handle, both algorithmically and computationally. Moreover, a technique to embed a discontinuous field in the extended virtual element discrete space is required whenever a mesh-independent modeling approach is preferred, such as in the simulation of cohesive fracture or when a finite element transitions from a continuous regime to a region with discontinuous kinematics [41].

\section{Numerical implementation}

In this section, we outline the main implementation aspects of the extended virtual element method introduced in Sections $3.3-3.5$. For the (nonenriched) virtual element formulation presented in Section 3.2, the interested reader can refer to [18].

\subsection{Fully enriched elements with singular fields}

To begin with, we assume a fully enriched element $E$, i.e., an element in which all the $N_{E}$ nodes are enriched with the two singular fields (15). Therefore, on such element we have $4 N_{E}$ 
degrees of freedom, and we can represent any virtual displacement field $\boldsymbol{v}_{X}^{h} \in \boldsymbol{V}_{X}^{h}(E)$ in terms of the shape functions of $\boldsymbol{V}_{X}^{h}(E)$ as $\boldsymbol{v}_{X}^{h}=\boldsymbol{N}_{X} \operatorname{dofs}\left(\boldsymbol{v}_{X}^{h}\right)$ where $\operatorname{dofs}\left(\boldsymbol{v}_{X}^{h}\right) \in \mathbb{R}^{4 N_{E}}$ is the vector of the degrees of freedom of $\boldsymbol{v}_{X}^{h}$ with respect to the basis function $\left\{\boldsymbol{\varphi}_{i}\right\}_{i=1}^{4 N_{E}}$ spanning $\boldsymbol{V}_{X}^{h}(E)$ and $N_{X} \in \mathbb{R}^{2 \times 4 N_{E}}$ is the matrix whose columns contain such basis functions

$$
N_{X} \equiv\left[\begin{array}{ccccccccc}
\varphi_{1} & 0 & \ldots & \check{u}_{x}^{I} \varphi_{1} & \check{u}_{x}^{I} \varphi_{2} & \ldots & \check{u}_{x}^{I I} \varphi_{1} & \check{u}_{x}^{I I} \varphi_{2} & \ldots \\
0 & \varphi_{1} & \ldots & \check{u}_{y}^{I} \varphi_{1} & \check{u}_{y}^{I} \varphi_{2} & \ldots & \check{u}_{x}^{I I} \varphi_{1} & \check{u}_{y}^{I I} \varphi_{2} & \ldots
\end{array}\right]=\left[\begin{array}{llll}
\varphi_{1} & \varphi_{2} & \ldots & \varphi_{4 N_{E}}
\end{array}\right] .
$$

Now, we define matrix $\boldsymbol{M}_{X} \in \mathbb{R}^{2 \times 8}$, whose columns are the basis vectors $\boldsymbol{m}_{\alpha}$ of $\mathbb{P}_{X}(E)$ introduced in 22 )

$$
\boldsymbol{M}_{X} \equiv\left[\begin{array}{cccccccc}
1 & 0 & \eta & \xi & 0 & \eta & \check{u}_{x}^{I} & \check{u}_{x}^{I I} \\
0 & 1 & -\xi & 0 & \eta & \xi & \check{u}_{y}^{I} & \check{u}_{y}^{I I}
\end{array}\right]=\left[\begin{array}{llllllll}
\boldsymbol{m}_{1} & \boldsymbol{m}_{2} & \boldsymbol{m}_{3} & \boldsymbol{m}_{4} & \boldsymbol{m}_{5} & \boldsymbol{m}_{6} & \boldsymbol{m}_{7} & \boldsymbol{m}_{8}
\end{array}\right] .
$$

Hereafter, we conveniently use the notation $\boldsymbol{m}_{7}=\check{\boldsymbol{u}}^{I}$ and $\boldsymbol{m}_{8}=\check{\boldsymbol{u}}^{I I}$. We represent the action of the projection operator $\Pi_{X}^{a}$ on the virtual basis functions by means of a matrix $\Pi_{X}^{a} \in \mathbb{R}^{8 \times 4 N_{E}}$. The $i$-th column of this matrix, denoted by $\pi^{i}=\left(\pi_{\alpha}^{i}\right) \in \mathbb{R}^{8}$, contains the coefficients of $\Pi_{X}^{a}\left(\varphi_{i}\right)$ when the projection is expanded on the basis $\boldsymbol{M}_{X}$ so that

$$
\Pi_{X}^{a}\left(\boldsymbol{\varphi}_{i}\right)=\boldsymbol{M}_{X} \boldsymbol{\pi}^{i}=\sum_{\alpha=1}^{8} \boldsymbol{m}_{\alpha} \pi_{\alpha}^{i}
$$

which in compact form can be expressed as $\Pi_{X}^{a}\left(\boldsymbol{N}_{X}\right)=\boldsymbol{M}_{X} \boldsymbol{\Pi}_{X}^{a}$.

We preliminarily observe that for every $\boldsymbol{q}_{X} \in \mathbb{P}_{X}(E)$ and every $\boldsymbol{v}_{X}^{h} \in \boldsymbol{V}_{X}^{h}(E)$, recalling that $\nabla \cdot \sigma\left(\boldsymbol{q}_{X}\right)=0$ and applying the divergence theorem, we find that

$$
\begin{aligned}
a^{E}\left(\boldsymbol{q}_{X}, \boldsymbol{v}_{X}^{h}\right) & =\int_{E} \boldsymbol{\sigma}\left(\boldsymbol{q}_{X}\right): \boldsymbol{\varepsilon}\left(\boldsymbol{v}_{X}^{h}\right) d \boldsymbol{x}=\int_{E} \boldsymbol{\sigma}\left(\boldsymbol{q}_{X}\right): \nabla \boldsymbol{v}_{X}^{h} d \boldsymbol{x} \\
& =\int_{E} \nabla \cdot\left(\boldsymbol{\sigma}\left(\boldsymbol{q}_{X}\right) \cdot \boldsymbol{v}_{X}^{h}\right)-\boldsymbol{v}_{X}^{h} \cdot\left(\nabla \cdot \boldsymbol{\sigma}\left(\boldsymbol{q}_{X}\right)\right) d \boldsymbol{x} \\
& =\int_{\partial E}\left(\boldsymbol{\sigma}\left(\boldsymbol{q}_{X}\right) \cdot \boldsymbol{v}_{X}^{h}\right) \cdot \boldsymbol{n}_{E} d s
\end{aligned}
$$

The boundary integral is always computable, since the integrand is known on the boundary.

By virtue of (36) and recalling the definition of the elliptic projection operator, we compute the projections of the virtual shape functions in terms of the basis of $\mathbb{P}_{X}(E)$. Indeed, for every $\boldsymbol{v}_{X}^{h} \in \boldsymbol{V}_{X}^{h}(E)$ we can write the following orthogonality condition:

$$
a^{E}\left(\boldsymbol{m}_{\beta}, \Pi_{X}^{a}\left(\boldsymbol{v}_{X}^{h}\right)\right)=a^{E}\left(\boldsymbol{m}_{\beta}, \boldsymbol{v}_{X}^{h}\right) \quad \beta=1, \ldots, 8 .
$$

Then, recall that $\operatorname{dofs}\left(v_{X}^{h}\right)=\left(v_{X, i}^{h}\right)$ are the $4 N_{E}$ degrees of freedom of $\boldsymbol{v}_{X}^{h}$ with respect to the basis $\left\{\boldsymbol{\varphi}_{i}\right\}_{i=1}^{4 N_{E}}$. In view of (35) and noting that $\boldsymbol{v}_{X}^{h}$ and $\operatorname{dofs}\left(\boldsymbol{v}_{X}^{h}\right)$ are arbitrary, we find that

$$
\begin{aligned}
& \sum_{i=1}^{4 N_{E}} a^{E}\left(\boldsymbol{m}_{\beta}, \Pi_{X}^{a}\left(\boldsymbol{\varphi}_{i}\right)\right) v_{X, i}^{h}=\sum_{i=1}^{4 N_{E}} a^{E}\left(\boldsymbol{m}_{\beta}, \boldsymbol{\varphi}_{i}\right) v_{X, i}^{h} \quad \beta=1, \ldots, 8 \\
& \Longrightarrow \sum_{\alpha=1}^{8} a^{E}\left(\boldsymbol{m}_{\beta}, \boldsymbol{m}_{\alpha}\right) \pi_{\alpha}^{i}=a^{E}\left(\boldsymbol{m}_{\beta}, \boldsymbol{\varphi}_{i}\right) \quad \beta=1, \ldots, 8, i=1, \ldots, 4 N_{E} \\
& \Longrightarrow \widehat{\boldsymbol{G}}_{X} \boldsymbol{\Pi}_{X}^{a}=\widehat{\boldsymbol{B}}_{X},
\end{aligned}
$$


where the companion matrices $\widehat{\boldsymbol{G}}_{X} \in \mathbb{R}^{8 \times 8}$ and $\widehat{\boldsymbol{B}}_{X} \in \mathbb{R}^{8 \times 4 N_{E}}$ are defined componentwise as

$$
\begin{aligned}
\left(\widehat{G}_{X}\right)_{\beta, \alpha} & =a^{E}\left(\boldsymbol{m}_{\beta}, \boldsymbol{m}_{\alpha}\right), \quad \beta, \alpha=1, \ldots, 8, \\
\left(\widehat{B}_{X}\right)_{\beta, i} & =a^{E}\left(\boldsymbol{m}_{\beta}, \boldsymbol{\varphi}_{i}\right), \quad \beta=1, \ldots, 8, \quad i=1, \ldots, 4 N_{E},
\end{aligned}
$$

or in the equivalent compact form by

$$
\widehat{\boldsymbol{G}}_{X}=a\left(\boldsymbol{M}_{X}^{T}, \boldsymbol{M}_{X}\right) \quad \text { and } \quad \widehat{\boldsymbol{B}}_{X}=a\left(\boldsymbol{M}_{X}^{T}, \boldsymbol{N}_{X}\right) .
$$

Recalling (36), both matrices $\widehat{\boldsymbol{G}}_{X}$ and $\widehat{\boldsymbol{B}}_{X}$ can be computed by integrating on the element boundary as follows:

$$
\begin{aligned}
\left(\widehat{G}_{X}\right)_{\beta, \alpha} & =\int_{E} \boldsymbol{\sigma}\left(\boldsymbol{m}_{\beta}\right): \boldsymbol{\varepsilon}\left(\boldsymbol{m}_{\alpha}\right) d \Omega=\int_{\partial E}\left(\boldsymbol{\sigma}\left(\boldsymbol{m}_{\beta}\right) \cdot \boldsymbol{m}_{\alpha}\right) \cdot \boldsymbol{n}_{E} d \Gamma \\
\left(\widehat{B}_{X}\right)_{\beta, i} & =\int_{E} \boldsymbol{\sigma}\left(\boldsymbol{m}_{\beta}\right): \boldsymbol{\varepsilon}\left(\boldsymbol{\varphi}_{i}\right) d \Omega=\int_{\partial E}\left(\boldsymbol{\sigma}\left(\boldsymbol{m}_{\beta}\right) \cdot \boldsymbol{\varphi}_{i}\right) \cdot \boldsymbol{n}_{E} d \Gamma
\end{aligned}
$$

The first three rows of $\widehat{\boldsymbol{G}}_{X}$ and $\widehat{\boldsymbol{B}}_{X}$ are zero, since the small strain tensor associated to rigid body motions is zero, and therefore $\widehat{\boldsymbol{G}}_{X}$ is rank deficient. To overcome this issue we use conditions (23b)-(23c), which imposes that the projector preserves the average nodal translations and rotations. So, we define the matrices $\boldsymbol{G}_{X}=\left(\left(G_{X}\right)_{\beta, \alpha}\right)$ and $\boldsymbol{B}_{X}=\left(\left(B_{X}\right)_{\beta, i}\right)$ as

$$
\left(G_{X}\right)_{\beta, \alpha}= \begin{cases}\frac{1}{N_{E}} \sum_{j=1}^{N_{E}} \boldsymbol{m}_{\alpha}\left(\boldsymbol{v}_{j}\right) & \beta=1,2, \\ \frac{1}{N_{E}} \sum_{j=1}^{N_{E}} \boldsymbol{r}\left(\boldsymbol{v}_{j}\right) \cdot \boldsymbol{m}_{\alpha}\left(\boldsymbol{v}_{j}\right) & \beta=3, \\ \left(\widehat{G}_{X}\right)_{\beta, \alpha} & \beta=4, \ldots, 8,\end{cases}
$$

and

$$
\left(B_{X}\right)_{\beta, i}= \begin{cases}\frac{1}{N_{E}} \sum_{j=1}^{N_{E}} \boldsymbol{\varphi}_{i}\left(\boldsymbol{v}_{j}\right) & \beta=1,2, \\ \frac{1}{N_{E}} \sum_{j=1}^{N_{E}} \boldsymbol{r}\left(\boldsymbol{v}_{j}\right) \cdot \boldsymbol{\varphi}_{i}\left(\boldsymbol{v}_{j}\right) & \beta=3, \\ \left(\widehat{B}_{X}\right)_{\beta, i} & \beta=4, \ldots, 4 N_{E} .\end{cases}
$$

Since matrix $\boldsymbol{G}_{X}$ is nonsingular, the projection matrix $\boldsymbol{\Pi}_{X}^{a}$ is the unique solution of the linear system $\boldsymbol{G}_{X} \boldsymbol{\Pi}_{X}^{a}=\boldsymbol{B}_{X}$. To derive the representation of the operator $\Pi_{X}^{a}$ with respect to the basis $\left\{\boldsymbol{\varphi}_{i}\right\}_{i=1}^{4 N_{E}}$ spanning $\boldsymbol{V}_{X}^{h}(E)$ we introduce matrix $\boldsymbol{D}_{X} \in \mathbb{R}^{4 N_{E} \times 8}$, whose $\alpha$-th column $(\alpha=1, \ldots, 8)$ contains the degrees of freedom of the vector polynomial $\boldsymbol{m}_{\alpha}$. Therefore, it holds that $\boldsymbol{M}_{X}=$ $N_{X} D_{X}$ and

$$
\Pi_{X}^{a}\left(\boldsymbol{N}_{X}\right)=\boldsymbol{M}_{X} \boldsymbol{\Pi}_{X}^{a}=\boldsymbol{N}_{X}\left(\boldsymbol{D}_{X} \boldsymbol{\Pi}_{X}^{a}\right)
$$


from which we infer that such matrix representation is given by matrix $\boldsymbol{D}_{X} \boldsymbol{\Pi}_{X}^{a}$. A straightforward calculation yields that $\boldsymbol{G}_{X}=\boldsymbol{B}_{X} \boldsymbol{D}_{X}$ in the X-VEM, which is similar in form to the standard relation in the VEM, $\boldsymbol{G}=\boldsymbol{B} \boldsymbol{D}$ [42]. This provides a means to verify the correctness of the computation of these matrices.

The stiffness matrix is given by the sum of a consistency and stability term,

$$
\boldsymbol{K}_{X}^{E}=\boldsymbol{K}_{X, c}^{E}+\boldsymbol{K}_{X, s}^{E}
$$

so that we can evaluate the extended stiffness bilinear form applied to $\boldsymbol{v}_{X}^{h}, \boldsymbol{w}_{X}^{h} \in \boldsymbol{V}_{X}^{h}(E)$ by using the degrees of freedom $\operatorname{dofs}\left(\boldsymbol{v}_{X}^{h}\right)$ and $\operatorname{dofs}\left(\boldsymbol{w}_{X}^{h}\right)$ as follows

$$
a_{X}^{h, E}\left(\boldsymbol{v}_{X}^{h}, \boldsymbol{w}_{X}^{h}\right)=\left(\operatorname{dofs}\left(\boldsymbol{v}_{X}^{h}\right)\right)^{T} \boldsymbol{K}_{X}^{E} \operatorname{dofs}\left(\boldsymbol{w}_{X}^{h}\right) .
$$

For every $\boldsymbol{v}_{X}^{h} \in \boldsymbol{V}_{X}^{h}(E)$, we first consider the relations

$$
\Pi_{X}^{a}\left(v_{X}^{h}\right)=\Pi_{X}^{a}\left(N_{X} \operatorname{dofs}\left(v_{X}^{h}\right)\right)=\Pi_{X}^{a}\left(N_{X}\right) \operatorname{dofs}\left(v_{X}^{h}\right)=M_{X} \Pi_{X}^{a} \operatorname{dofs}\left(v_{X}^{h}\right)=\left(\operatorname{dofs}\left(v_{X}^{h}\right)\right)^{T}\left(\Pi_{X}^{a}\right)^{T}\left(M_{X}\right)^{T} .
$$

Recalling (44), we compute the consistency term as

$$
\begin{aligned}
a^{E}\left(\Pi_{X}^{a}\left(\boldsymbol{v}_{X}^{h}\right), \Pi_{X}^{a}\left(\boldsymbol{w}_{X}^{h}\right)\right) & =\left(\boldsymbol{d o f s}\left(\boldsymbol{w}_{X}^{h}\right)\right)^{T}\left(\boldsymbol{\Pi}_{X}^{a}\right)^{T} a^{E}\left(\boldsymbol{M}_{X}^{T}, \boldsymbol{M}_{X}\right) \boldsymbol{\Pi}_{X}^{a} \operatorname{dofs}\left(\boldsymbol{v}_{X}^{h}\right) \\
& =\left(\boldsymbol{d o f s}\left(\boldsymbol{w}_{X}^{h}\right)\right)^{T}\left(\boldsymbol{\Pi}_{X}^{a}\right)^{T} \widehat{\boldsymbol{G}}_{X} \boldsymbol{\Pi}_{X}^{a} \mathbf{d o f s}\left(\boldsymbol{w}_{X}^{h}\right) .
\end{aligned}
$$

By comparison, we see that

$$
\boldsymbol{K}_{X, c}^{E}=\left(\boldsymbol{\Pi}_{X}^{a}\right)^{T} \widehat{\boldsymbol{G}}_{X} \boldsymbol{\Pi}_{X}^{a} .
$$

For the stability term we generalize the so-called dofi-dofi [12] and D-recipe [43] stabilizations by evaluating the second term in (24) at the element vertices. To this end, we first note that

$$
\boldsymbol{v}_{X}^{h}\left(\boldsymbol{x}_{\ell}\right)-\Pi_{X}^{a}\left(\boldsymbol{v}_{X}^{h}\right)\left(\boldsymbol{x}_{\ell}\right)=\boldsymbol{N}_{X}\left(\boldsymbol{x}_{\ell}\right) \mathbf{d o f} \mathbf{s}\left(\boldsymbol{w}_{X}^{h}\right)-\boldsymbol{N}_{X}\left(\boldsymbol{x}_{\ell}\right) \boldsymbol{D}_{X} \Pi_{X}^{a} \mathbf{d o f} \mathbf{s}\left(\boldsymbol{w}_{X}^{h}\right) .
$$

Definition (33) and $\varphi_{j}\left(\boldsymbol{x}_{\ell}\right)=\delta_{j \ell}$ implies that

$$
\begin{aligned}
j \text {-th column of } \boldsymbol{N}_{X}\left(\boldsymbol{x}_{\ell}\right)= & \begin{array}{ll}
\left(\varphi_{j}\left(\boldsymbol{x}_{\ell}\right), 0\right)^{T} & \text { for } 1 \leq j \leq 2 N_{E}, j \text { odd, } \\
\left(0, \varphi_{j}\left(\boldsymbol{x}_{\ell}\right)\right)^{T} & \text { for } 1 \leq j \leq 2 N_{E}, j \text { even, } \\
\left(\check{u}_{x}^{I}\left(\boldsymbol{x}_{\ell}\right) \phi_{j}\left(\boldsymbol{x}_{\ell}\right), \check{u}_{y}^{I}\left(\boldsymbol{x}_{\ell}\right) \varphi_{j}\left(\boldsymbol{x}_{\ell}\right)\right)^{T} & \text { for } 1+2 N_{E} \leq j \leq 3 N_{E}, \\
\left(\check{u}_{x}^{I I}\left(\boldsymbol{x}_{\ell}\right) \phi_{j}\left(\boldsymbol{x}_{\ell}\right), \check{u}_{y}^{I I}\left(\boldsymbol{x}_{\ell}\right) \varphi_{j}\left(\boldsymbol{x}_{\ell}\right)\right)^{T} & \text { for } 1+3 N_{E} \leq j \leq 4 N_{E},
\end{array} \\
& = \begin{cases}\left(\delta_{j, \ell}, 0\right)^{T} & \text { for } 1 \leq j \leq 2 N_{E}, j \text { odd }, \\
\left(0, \delta_{j, \ell}\right)^{T} & \text { for } 1 \leq j \leq 2 N_{E}, j \text { even, } \\
\left(\check{u}_{x}^{I}\left(\boldsymbol{x}_{\ell}\right) \delta_{j, \ell}, \check{u}_{y}^{I}\left(\boldsymbol{x}_{\ell}\right) \delta_{j, \ell}\right)^{T} & \text { for } 1+2 N_{E} \leq j \leq 3 N_{E}, \\
\left(\check{u}_{x}^{I I}\left(\boldsymbol{x}_{\ell}\right) \delta_{j, \ell}, \check{u}_{y}^{I I}\left(\boldsymbol{x}_{\ell}\right) \delta_{j, \ell}\right)^{T} & \text { for } 1+3 N_{E} \leq j \leq 4 N_{E},\end{cases}
\end{aligned}
$$


for $\ell=1, \ldots, N_{E}$. We collect $N_{X}\left(\boldsymbol{x}_{\ell}\right)$ in the compact block-diagonal matrix $\boldsymbol{J}=\operatorname{diag}\left(\boldsymbol{J}_{11}, \boldsymbol{J}_{22}\right) \in$ $\mathbb{R}^{4 N_{E} \times 4 N_{E}}$ such that $\boldsymbol{J}_{11}=\boldsymbol{I}_{2 N_{E} \times 2 N_{E}}$, which is the $2 N_{E} \times 2 N_{E}$-sized identity matrix, and $\boldsymbol{J}_{22}=$ $\left[\boldsymbol{J}_{22}^{I}, \boldsymbol{J}_{22}^{I I}\right]$, with

$$
\begin{aligned}
& \boldsymbol{J}_{22}^{I}=\operatorname{diag}\left(\check{\boldsymbol{u}}^{I}\left(\boldsymbol{x}_{1}\right), \check{\boldsymbol{u}}^{I}\left(\boldsymbol{x}_{2}\right), \ldots, \check{\boldsymbol{u}}^{I}\left(\boldsymbol{x}_{N_{E}}\right)\right), \\
& \boldsymbol{J}_{22}^{I I}=\operatorname{diag}\left(\check{\boldsymbol{u}}^{I I}\left(\boldsymbol{x}_{1}\right), \check{\boldsymbol{u}}^{I I}\left(\boldsymbol{x}_{2}\right), \ldots, \check{\boldsymbol{u}}^{I I}\left(\boldsymbol{x}_{N_{E}}\right)\right) .
\end{aligned}
$$

Here, $\boldsymbol{J}_{11} \in \mathbb{R}^{2 N_{E} \times 2 N_{E}}$ collects the values of the first $2 N_{E}$ columns of $\boldsymbol{N}_{X}\left(\boldsymbol{x}_{\ell}\right)$ and is such that the $\ell$-th row corresponds to the $\ell$-th element vertex. In turn, the matrix blocks $\boldsymbol{J}_{22}^{I}$ and $\boldsymbol{J}_{22}^{I I}$ are $2 N_{E} \times N_{E}$-sized matrices that collect the $N_{E}$ columns of $N_{X}$ corresponding to $\boldsymbol{\psi}^{I} \boldsymbol{V}^{h, *}(E)$ and $\psi^{I I} V^{h, *}(E)$, and again each row corresponds to a given element vertex. Finally, we introduce the matrix $\widehat{\boldsymbol{D}}_{X}=\boldsymbol{J} \boldsymbol{D}_{X}$, and we write the dofi-dofi stabilization as

$$
\begin{array}{r}
S_{X}^{E}\left(\boldsymbol{v}_{X}^{h}-\Pi_{X}^{a}\left(\boldsymbol{v}_{X}^{h}\right), \boldsymbol{w}_{X}^{h}-\Pi_{X}^{a}\left(\boldsymbol{w}_{X}^{h}\right)\right)=\tau \sum_{\ell=1}^{N_{E}}\left(\boldsymbol{N}_{X}\left(\boldsymbol{x}_{\ell}\right) \operatorname{dofs}\left(\boldsymbol{v}_{X}^{h}\right)-\boldsymbol{N}_{X}\left(\boldsymbol{x}_{\ell}\right) \boldsymbol{D}_{X} \Pi_{X}^{a} \mathbf{d o f s}\left(\boldsymbol{v}_{X}^{h}\right)\right) \\
\times\left(\boldsymbol{N}_{X}\left(\boldsymbol{x}_{\ell}\right) \mathbf{d o f s}\left(\boldsymbol{w}_{X}^{h}\right)-\boldsymbol{N}_{X}\left(\boldsymbol{x}_{\ell}\right) \boldsymbol{D}_{X} \boldsymbol{\Pi}_{X}^{a} \mathbf{d o f s}\left(\boldsymbol{w}_{X}^{h}\right)\right) \\
=\tau\left(\operatorname{dofs}\left(\boldsymbol{v}_{X}^{h}\right)\right)^{T}\left(\boldsymbol{J}-\widehat{\boldsymbol{D}}_{X} \boldsymbol{\Pi}_{X}^{a}\right)^{T}\left(\boldsymbol{J}-\widehat{\boldsymbol{D}}_{X} \boldsymbol{\Pi}_{X}^{a}\right)\left(\operatorname{dofs}\left(\boldsymbol{w}_{X}^{h}\right)\right),
\end{array}
$$

where $\tau$ is a suitable scaling parameter; a possible choice is $\tau=\alpha \operatorname{trace}\left(\boldsymbol{K}_{X, c}^{E}\right) / 4 N_{E}$, where $\alpha$ is a constant (a sensitivity analysis on the choice of $\alpha$ is presented in the next section). Hence,

$$
\boldsymbol{K}_{X, s}^{E}=\tau\left(\boldsymbol{J}-\widehat{\boldsymbol{D}}_{X} \boldsymbol{\Pi}_{X}^{a}\right)^{T}\left(\boldsymbol{J}-\widehat{\boldsymbol{D}}_{X} \boldsymbol{\Pi}_{X}^{a}\right) .
$$

Similarly, the D-recipe stabilization, which was originally proposed in [43] for the Poisson equation, can be generalized by taking the stabilization matrix

$$
\boldsymbol{K}_{X, s}^{E}=\left(\boldsymbol{J}-\widehat{\boldsymbol{D}}_{X} \boldsymbol{\Pi}_{X}^{a}\right)^{T} \boldsymbol{S}_{X}\left(\boldsymbol{J}-\widehat{\boldsymbol{D}}_{X} \boldsymbol{\Pi}_{X}^{a}\right),
$$

where

$$
\left(\boldsymbol{S}_{X}\right)_{i j}=\delta_{i j} \max \left(\frac{\operatorname{trace}(\boldsymbol{C})}{3} h_{E},\left(\boldsymbol{K}_{X, c}^{E}\right)_{i i}\right) \quad i, j=1, \ldots, N_{E}
$$

and with the same definition of $\boldsymbol{J}$ and $\widehat{\boldsymbol{D}}_{X}$ introduced above.

\subsection{Partially enriched elements with singular fields}

Let $\boldsymbol{E}_{X} \in \mathbb{R}^{4 N_{E} \times\left(2 N_{E}+2 k_{E}\right)}$ be the matrix representation of the extension operator $\mathcal{E}_{k_{E}}$ introduced in Section 3.4, so that every vector-valued field $\widetilde{\boldsymbol{v}}_{X}^{h} \in \widetilde{\boldsymbol{V}}_{X}^{h}(E)$ with degrees of freedom $\operatorname{dofs}\left(\widetilde{\boldsymbol{v}}_{X}^{h}\right) \in \mathbb{R}^{2 N_{E}+2 k_{E}}$ is remapped into the vector-valued field $\boldsymbol{v}_{X}^{h} \in \boldsymbol{V}_{X}^{h}(E)$ with degrees of free$\operatorname{dom} \operatorname{dofs}\left(\boldsymbol{v}_{X}^{h}\right)=\boldsymbol{E}_{X} \operatorname{dofs}\left(\widetilde{\boldsymbol{v}}_{X}^{h}\right) \in \mathbb{R}^{4 N_{E}}$. Let $\widetilde{\boldsymbol{K}}_{X}^{E} \in \mathbb{R}^{\left(2 N_{E}+2 k_{E}\right) \times\left(2 N_{E}+2 k_{E}\right)}$ such that

$$
\widetilde{a}_{X}^{h, E}\left(\widetilde{\boldsymbol{v}}_{X}^{h}, \widetilde{\boldsymbol{w}}_{X}^{h}\right)=\left(\operatorname{dofs}\left(\widetilde{\boldsymbol{v}}_{X}^{h}\right)\right)^{T} \widetilde{\boldsymbol{K}}_{X}^{E} \operatorname{dofs}\left(\widetilde{\boldsymbol{w}}_{X}^{h}\right) .
$$


Now, starting from definition (29) and using (47), a straightforward calculation yields

$$
\begin{aligned}
\widetilde{a}_{X}^{h, E}\left(\widetilde{\boldsymbol{v}}_{X}^{h}, \widetilde{\boldsymbol{w}}_{X}^{h}\right) & =a_{X}^{h, E}\left(\mathcal{E}_{k_{E}} \boldsymbol{v}_{X}^{h}, \mathcal{E}_{k_{E}} \widetilde{\boldsymbol{w}}_{X}^{h}\right)=\left(\operatorname{dofs}\left(\mathcal{E}_{k_{E}} \boldsymbol{w}_{X}^{h}\right)\right)^{T} \boldsymbol{K}_{X}^{E} \operatorname{dofs}\left(\mathcal{E}_{k_{E}} \boldsymbol{v}_{X}^{h}\right) \\
& =\left(\boldsymbol{E}_{X} \operatorname{dofs}\left(\widetilde{\boldsymbol{w}}_{X}^{h}\right)\right)^{T} \boldsymbol{K}_{X}^{E} \boldsymbol{E}_{X} \operatorname{dofs}\left(\widetilde{\boldsymbol{v}}_{X}^{h}\right)=\left(\operatorname{dofs}\left(\widetilde{\boldsymbol{w}}_{X}^{h}\right)\right)^{T}\left(\boldsymbol{E}_{X}\right)^{T} \boldsymbol{K}_{X}^{E} \boldsymbol{E}_{X} \operatorname{dofs}\left(\widetilde{\boldsymbol{v}}_{X}^{h}\right),
\end{aligned}
$$

and by comparison with (47) it follows that

$$
\widetilde{\boldsymbol{K}}_{X}^{E}=\left(\boldsymbol{E}_{X}\right)^{T} \boldsymbol{K}_{X}^{E} \boldsymbol{E}_{X}
$$

since $\widetilde{\boldsymbol{v}}_{X}^{h}$ and $\widetilde{\boldsymbol{w}}_{X}^{h}$ are arbitrary. To conclude this section, we are only left to explain the construction of the matrix $\boldsymbol{E}_{X}$ that embodies definition (28). To obtain such a matrix, we take the $4 N_{E} \times 4 N_{E}$-size identity matrix and remove the $2\left(N_{E}-k_{E}\right)$ columns that corresponds to the basis functions of the nonenriched vertices in $\psi^{I} V^{h, *}(E) \oplus \boldsymbol{\psi}^{I I} \boldsymbol{V}^{h, *}(E)$. Finally we note that when we apply $\left(\boldsymbol{E}_{X}\right)^{T}$ to the left of matrix $\boldsymbol{K}_{X}^{E}$ and $\boldsymbol{E}_{X}$ to the right of matrix $\boldsymbol{K}_{X}^{E}$ we are indeed selecting the rows and the columns of $\boldsymbol{K}_{X}^{E}$ that corresponds to all the degrees of freedom of $\boldsymbol{V}^{h}(E)$ and the degrees of freedom of the enriched nodes of $\boldsymbol{\psi}^{I} \boldsymbol{V}^{h, *}(E) \oplus \boldsymbol{\psi}^{I I} \boldsymbol{V}^{h, *}(E)$. In the numerical implementation we do not need to construct the matrix $\boldsymbol{E}_{X}$ explicitly and compute $\widetilde{\boldsymbol{K}}_{X}^{E}$ using (48), since we can simply build the stiffness matrix $\boldsymbol{K}_{X}^{E}$ of the full enrichment case and remove all rows and columns that refer to non-enriched nodal degrees of freedom.

\subsection{Embedding discontinuities}

Let us consider the case in which an element $E$, fully enriched according to the construction outlined in Section 4.1, is also cut by a crack $\gamma$ into two subelements $E^{-}$and $E^{+}$, see Fig. 3 . Then, following the approach presented in Section 3.5, we can compute the projectors $\Pi_{X}^{a-}$ and

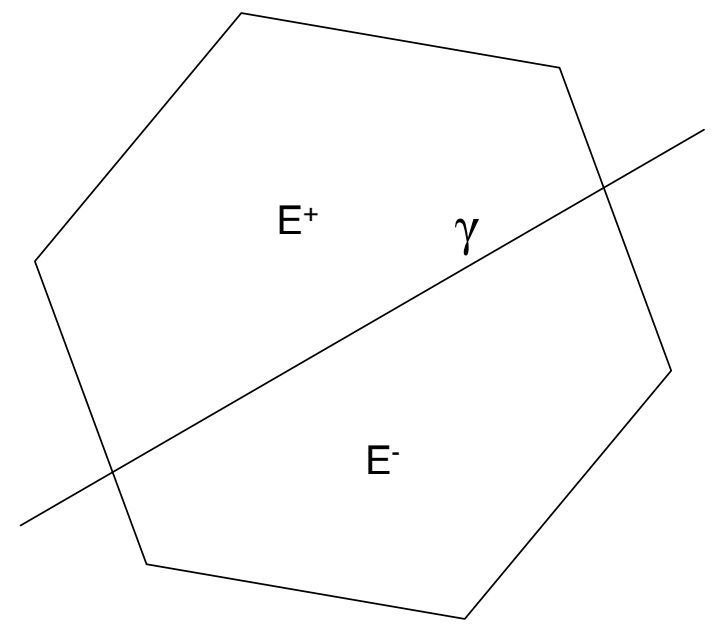

Figure 3: Crack line $\gamma$ cuts element $E$ into two subelements $E^{-}$and $E^{+}$.

$\Pi_{X}^{a+}$ on the two subelements $E^{-}$and $E^{+}$generated by the crack line $\gamma$ intersecting element $E$. Recalling that, in the present approach, the number of degrees of freedom is doubled and is equal to $N_{\mathrm{dof}}^{\mathrm{HH}}=8 N_{E}$, we denote by $i^{-}$and $i^{+}$the degrees of freedom associated to $\boldsymbol{\phi}_{i}^{-}$and $\boldsymbol{\phi}_{i}^{+}$ respectively, with $i=1, \ldots, 4 N_{E}$. 
Then, the consistent part of the stiffness matrix has a diagonal block structure and is composed by the following submatrix blocks:

$$
\begin{aligned}
& \left(\boldsymbol{K}_{X, c}^{E}\right)_{+,+}=\left(\boldsymbol{\Pi}_{X}^{a+}\right)^{T} \widehat{\boldsymbol{G}}_{X}^{+} \boldsymbol{\Pi}_{X}^{a+}, \\
& \left(\boldsymbol{K}_{X, c}^{E}\right)_{-,-}=\left(\boldsymbol{\Pi}_{X}^{a-}\right)^{T} \widehat{\boldsymbol{G}}_{X}^{-} \boldsymbol{\Pi}_{X}^{a-},
\end{aligned}
$$

where matrices $\widehat{\boldsymbol{G}}_{X}^{-}$and $\widehat{\boldsymbol{G}}_{X}^{+}$are the counterparts of $\widehat{\boldsymbol{G}}_{X}$ computed for $E^{-}$and $E^{+}$, respectively.

On the other hand, the general expression for the stabilization part shares the same diagonal block structures and reads:

$$
\begin{aligned}
& \left(\boldsymbol{K}_{X, s}^{E}\right)_{+,+}=\tau\left(\boldsymbol{J}-\widehat{\boldsymbol{D}}_{X}^{+} \boldsymbol{\Pi}_{X}^{a+}\right)^{T}\left(\boldsymbol{J}-\widehat{\boldsymbol{D}}_{X}^{+} \boldsymbol{\Pi}_{X}^{a+}\right), \\
& \left(\boldsymbol{K}_{X, s}^{E}\right)_{-,-}=\tau\left(\boldsymbol{J}-\widehat{\boldsymbol{D}}_{X}^{-} \boldsymbol{\Pi}_{X}^{a-}\right)^{T}\left(\boldsymbol{J}-\widehat{\boldsymbol{D}}_{X}^{-} \boldsymbol{\Pi}_{X}^{a-}\right),
\end{aligned}
$$

where matrices $\widehat{\boldsymbol{D}}_{X}^{-}$and $\widehat{\boldsymbol{D}}_{X}^{+}$are the counterparts of $\widehat{\boldsymbol{D}}_{X}$ computed for $E^{-}$and $E^{+}$, respectively.

\subsection{Computation of stress intensity factors}

In order to determine the susceptibility of a given elastic two-dimensional body to fracture growth we need to extract appropriate crack tip parameters such as the $J$-integral and mixedmode stress intensity factors. We consider a neighborhood of the crack tip, as shown in Fig. 4a. Given an arbitrary closed path $\Gamma$ around the crack tip, the $J$-integral is defined as:

$$
J=\int_{\Gamma}\left(W d x_{2}-T_{i} \frac{\partial u_{i}}{\partial x_{1}} d s\right)
$$

which is path independent under the assumptions of small deformations, elastic material behavior and quasi-statically applied loads [44]. In (51), $u_{i}$ is the $i$-th component of the displacement field, $d s$ is the differential of the arc length of $\Gamma, T_{i}$ is the $i$-th component of the traction vector along $\Gamma$ and $W$ is the strain energy density, which is defined as:

$$
W=\int_{0}^{\varepsilon_{i j}} \sigma_{i j} d \varepsilon_{i j}=\frac{1}{2} \sigma_{i j} \varepsilon_{i j}
$$

However, the $J$-integral in (51) is not well-suited for numerical computations, since it is defined on a vanishingly small closed path. For this reason, in numerical procedures, Li et al. [45] proposed to recast the line integral (51) into a domain integral over an annular region $\Omega$, bounded by an inner closed curve $\partial \Omega_{i} \rightarrow 0$, which contains the crack tip, and an outer closed curve $\partial \Omega_{o}$ :

$$
J=\int_{\Omega}\left(\sigma_{i j} \frac{\partial u_{i}}{\partial x_{1}}-W \delta_{1 i}\right) \frac{\partial w}{\partial x_{i}} d \Omega
$$

where $w$ is a suitable weight function that is equal to unity within the domain bounded by $\partial \Omega_{o}$ and vanishes on $\partial \Omega_{o}$. Based on this definition, given two equilibrium states denoted by superscripts (1) and (2), referred to as the present state and an auxiliary state respectively, the interaction integral in domain form is given by

$$
I^{(1,2)}=\int_{\Omega} F_{j}\left(x_{1}, x_{2}\right) \frac{\partial w}{\partial x_{j}} d \Omega
$$




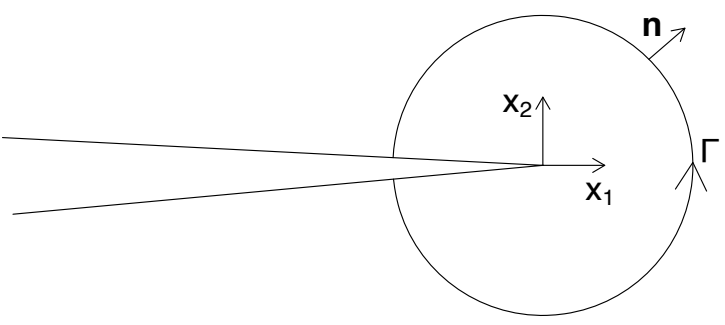

(a)

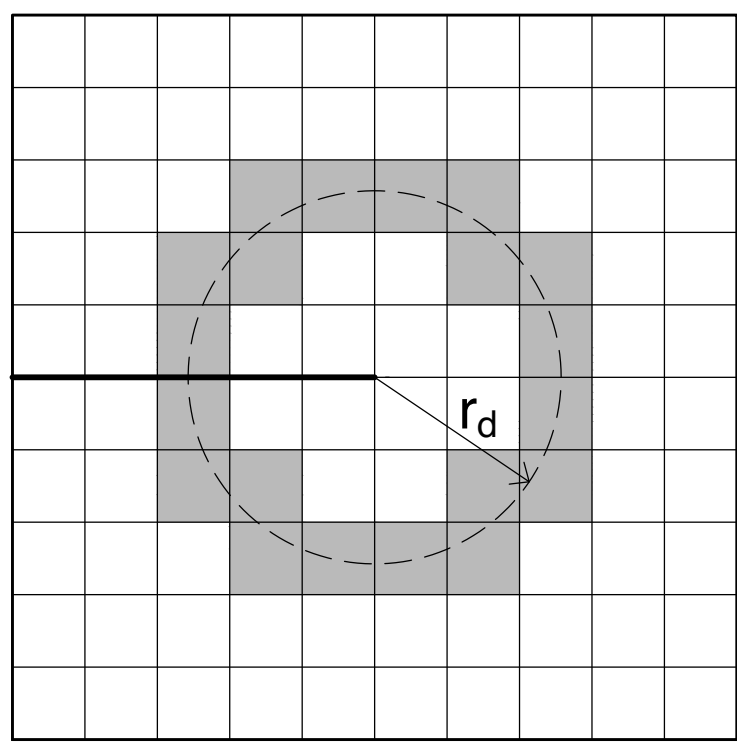

(b)

Figure 4: Local crack tip coordinates (a) and discretized J-domain (shaded area) (b).

where

$$
F_{j}\left(x_{1}, x_{2}\right)=\sigma_{i j}^{(1)} \frac{\partial u_{i}^{(2)}}{\partial x_{1}}+\sigma_{i j}^{(2)} \frac{\partial u_{i}^{(1)}}{\partial x_{1}}-W^{(1,2)} \delta_{1 j},
$$

and $W^{(1,2)}=\sigma_{i j}^{(1)} \varepsilon_{i j}^{(2)}=\sigma_{i j}^{(2)} \varepsilon_{i j}^{(1)}$ is the interaction strain energy.

The stress intensity factors for mode I and mode II crack opening, respectively denoted by $K_{I}$ and $K_{I I}$, are computed using the relation

$$
I^{(1,2)}=\frac{2}{E^{\prime}}\left[K_{I}^{(1)} K_{I}^{(2)}+K_{I I}^{(1)} K_{I I}^{(2)}\right]
$$

where $E^{\prime}=E$ for plane stress conditions and $E^{\prime}=E /\left(1-v^{2}\right)$ for plain strain conditions. Indeed, on choosing the auxiliary field corresponding to $K_{I}=1$ and $K_{I I}=0$ allows $K_{I}$ to be extracted in a straightforward manner and similarly on selecting the auxiliary field corresponding to $K_{I}=0$ and $K_{I I}=1$ allows $K_{I I}$ to be computed:

$$
K_{I}=\frac{E^{\prime}}{2} I^{(1, I)}, \quad K_{I I}=\frac{E^{\prime}}{2} I^{(1, I I)} .
$$


However, computing the interaction integral (54) is not straightforward in the X-VEM, since the numerical integration is performed over polygonal elements. For this reason, after considering a $J$-domain that is an annular region $\Omega_{J}$ that consists of a ring of elements that are intersected by a circle of given radius $r_{d}$ centered on the crack tip (i.e., the shaded area in Fig. 4b), we apply the divergence theorem and transform the domain integral (54) into a line integral that is evaluated on the boundaries of the element [29]:

$$
I^{(1,2)}=\sum_{E \in \Omega_{J}}\left(\int_{\partial E} F_{j}\left(x_{1}, x_{2}\right) w n_{j} d \Gamma-\int_{E} \frac{\partial F_{j}}{\partial x_{j}}\left(x_{1}, x_{2}\right) w d \Omega\right) .
$$

We note that $\nabla \cdot \boldsymbol{F}=0$ in $(58)$ since the auxiliary fields are equilibrated, and therefore only the boundary integral needs to be computed.

Since virtual shape functions are not known in the interior of the elements, we use the elliptic projection of the solution in terms of displacements to compute the corresponding deformation field and the stress components. Hence, the interaction integral can be finally computed as:

$$
I^{(1,2)}=\sum_{E \in \Omega_{J}} \int_{\partial E}\left[\sigma_{i j}\left(\Pi_{E}^{a}\left(u_{i}^{(1)}\right)\right) \frac{\partial u_{i}^{(2)}}{\partial x_{1}}+\sigma_{i j}^{(2)} \frac{\partial \Pi_{E}^{a}\left(u_{i}^{(1)}\right)}{\partial x_{1}}-\widetilde{W}^{(1,2)} \delta_{1 j}\right] w n_{j} d \Gamma
$$

where $\widetilde{W}^{(1,2)}=\sigma_{i j}\left(\Pi_{E}^{a}\left(u_{i}^{(1)}\right)\right) \varepsilon_{i j}^{(2)}$. From a computational viewpoint, it is convenient to assume the weight function $w$ to be equal to unity on all nodes in $\Omega_{J}$ that lie within the circle of radius $r_{d}$, and equal to zero on all nodes in $\Omega_{J}$ that lie outside the circle of radius $r_{d}$. Along element edges, where integrations are carried out, linear interpolation of $w$ between its nodal values is adopted.

\section{Numerical examples}

In order to check the consistency of the X-VEM, we first conduct two distinct patch tests: an extended patch test, addressing the enrichment with singular fields as described in Section 4.1 , and a discontinuous patch test aimed at assessing the inclusion of discontinuities in the discrete space by means of the approach presented in Section 3.5. Then, we test the X-VEM on a benchmark problem to establish the convergence rate of the method and the accuracy of the stress intensity factors. Unless stated otherwise, Young's modulus $E=10^{5}$ and Poisson ratio $v=0.3$ are chosen in the numerical computations.

\subsection{Extended patch test}

The extended patch test ensures that the singular enrichment fields in (15) can be exactly reproduced using the X-VEM. To perform the extended patch test, we consider a square elastic plate that occupies the region $(-1,1)^{2}$ under plane strain conditions, with a horizontal crack of unit length that extends from $(-1,0)$ to $(0,0)$ (see Fig. 5a).

Both a coarse mesh of $10 \times 10$ square elements and a coarse mesh of 64 polygonal elements are considered. For the purposes of the extended patch test, the crack is modeled explicitly so that we do not have to embed the discontinuity in the discrete space. All the nodes in the domain are enriched and the Cartesian components of the near-tip displacement fields (15) are imposed on the boundary of the domain by requiring that all the enriched boundary degrees of freedom 


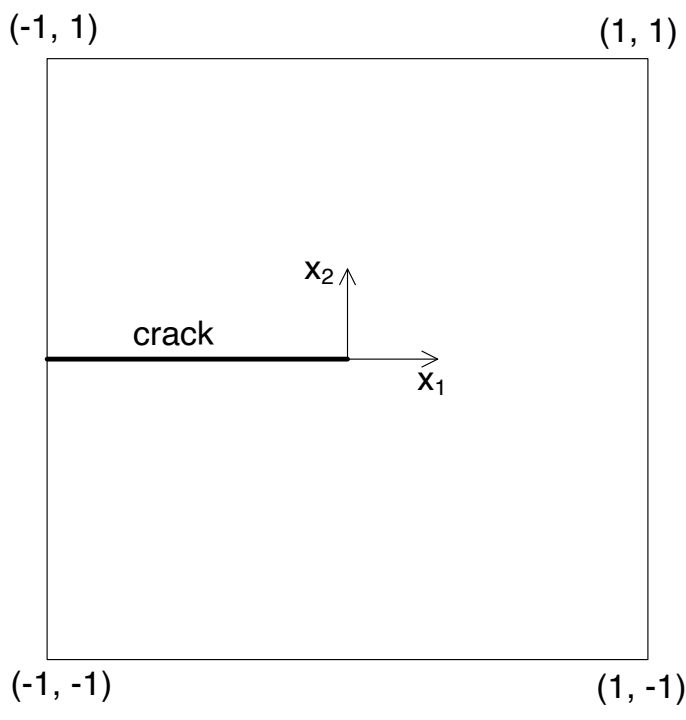

(a)

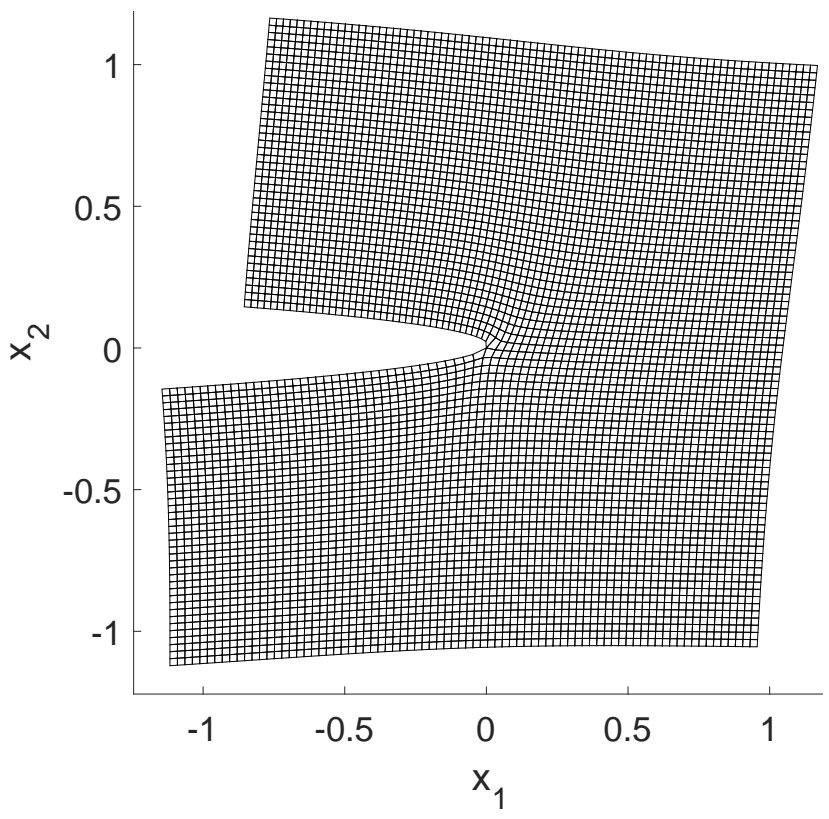

(b)

Figure 5: Mixed mode I and mode II crack opening benchmark problem: domain geometry (a) and exact deformed shape (b).

are equal to 1 and all the standard boundary degrees of freedom are equal to 0 . The exact displacement solution field is shown in Fig. 5b. As detailed in the previous section, integrals need to be evaluated over the element boundary only. We adopt a 16-points Gauss quadrature rule on each element edge.

As a measure for the error of the numerical solution with respect to the exact solution we adopted the relative error in strain energy, which is computed as

$$
E\left(\boldsymbol{u}^{h}\right)=\frac{\left|a(\tilde{\boldsymbol{u}}, \tilde{\boldsymbol{u}})-a\left(\boldsymbol{u}^{h}, \boldsymbol{u}^{h}\right)\right|}{a(\boldsymbol{u}, \boldsymbol{u})},
$$

where $\frac{1}{2} a(\boldsymbol{u}, \boldsymbol{u})=1.6776885579 \times 10^{-5}$ is the strain energy of the exact solution $\boldsymbol{u}$, and $\tilde{\boldsymbol{u}}^{h}$ is the projection of the discrete solution $\boldsymbol{u}^{h}$, which is defined as:

$$
\tilde{\boldsymbol{u}}^{h}=\sum_{E \in \mathcal{T}} \Pi_{E}^{a} \boldsymbol{u}^{h}
$$

We also adopt this error measure in the subsequent sections that follow. In (61), we use the same symbol $\Pi_{E}^{a}$ to denote the restriction of the virtual element functions defined on the element $E$ of the projection operator $\Pi^{a}$ if $E$ is a nonenriched element and the projection operator $\Pi_{X}^{a}$ if $E$ is an enriched element. The choice of using the projection $\tilde{\boldsymbol{u}}^{h}$ of the solution $\boldsymbol{u}^{h}$ follows from observing that it is not possible to compute the true energy associated with $\boldsymbol{u}^{h}$, since the virtual functions are not explicitly known [46]. The relative error in strain energy for the extended patch tests is provided in Table 1, which clearly shows that the X-VEM delivers sound accuracy in reproducing the enrichment fields, although the error is affected by numerical integration of singular functions. 
Table 1: Relative error in strain energy for the extended patch test on the $(-1,1) \times(1,1)$ square domain with horizontal crack.

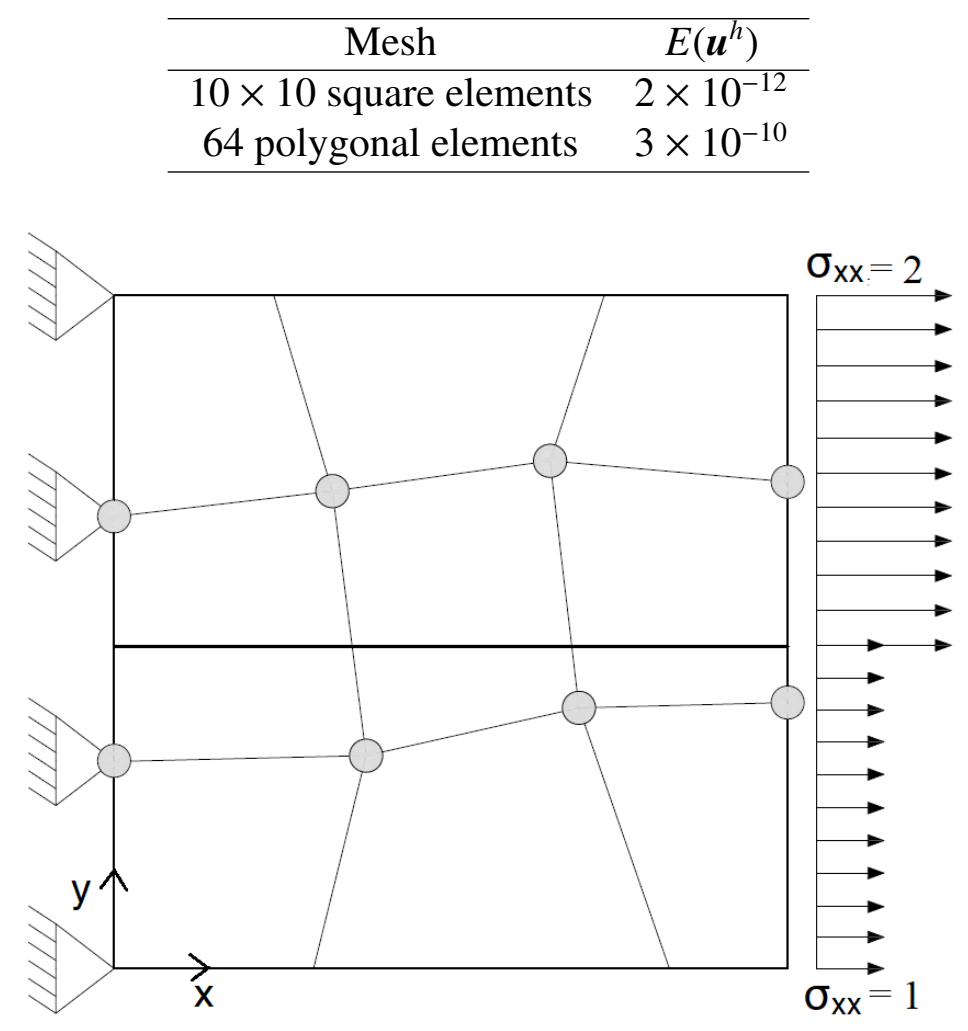

Figure 6: Geometry and loading conditions of the discontinuous patch test.

\subsection{Discontinuous patch test}

In order to evaluate the effectiveness and robustness of the X-VEM in the presence of discontinuities, formulated according to the approach presented in Section 3.5, we adopt a suitable patch test which entails solving a problem whose exact solution is discontinuous and lies in the discrete space. We then verify if the extended virtual element approximation matches such a solution. To this end, we here adapt the discontinuous patch test first proposed by Dolbow and Devan [47] in finite strain elasticity to the present context of plain strain linear elasticity. The test involves solving the problem of a $2 \mathrm{D}$ elastic domain occupying the unit square domain $\Omega=(0,1)^{2}$ that is bisected by an horizontal crack $\gamma$ into two open subdomains $\Omega^{-}=(0,1) \times(0,1 / 2)$ and $\Omega^{+}=(0,1) \times(1 / 2,1)$. The crack is implicitly included in the model following the construction proposed in Section 3.5. For the sake of simplicity, we assume $E=1$ and $v=0$, so that the problem is reduced to one dimension. As boundary conditions, we prescribe zero displacements along the edge $x=0$, a discontinuous distribution of horizontal tractions along the edge $x=1$ and zero tractions along the horizontal edges $y=0$ and $y=1$ :

$$
\begin{aligned}
& \boldsymbol{u}(0, y)=\mathbf{0}, \\
& \sigma_{x x}(1, y)=\left\{\begin{array}{ll}
1, & y \leq 1 / 2 \\
2, & y>1 / 2
\end{array}, \quad \sigma_{y y}(1, y)=\sigma_{x y}(1, y)=0,\right. \\
& \sigma_{y y}(x, 0)=\sigma_{x y}(x, 0)=0,
\end{aligned}
$$




$$
\sigma_{y y}(x, 1)=\sigma_{x y}(x, 1)=0 .
$$

For this problem, whose geometry and boundary conditions are depicted in Fig. 6, the exact solution is the following piecewise linear function

$$
\boldsymbol{u}(x, y)= \begin{cases}{[x, 0]^{T},} & (x, y) \in \Omega^{-}, \\ {[2 x, 0]^{T},} & (x, y) \in \Omega^{+} .\end{cases}
$$

The exact solution (63) belongs to the discrete space. In agreement with the expectations, the extended virtual element formulation presented in Section 3.5, which uses distinct projector operators on the two subdomains generated by the horizontal crack, passes the proposed patch test with a relative error in strain energy of $2 \times 10^{-13}$.

\subsection{Convergence study}

We study the convergence of the X-VEM for the problem of a two-dimensional square plate under plain strain conditions that contains a horizontal crack, extending from the boundary to the center of the specimen. The boundary conditions are such that mixed-mode conditions prevail. The geometry of the domain is the same adopted as that for the extended patch test in Section 5.1 and is shown in Fig. 5a. On the boundary of the domain, we apply the exact near-tip displacement fields (14), which are also employed as enrichment fields for the X-VEM and represent the exact solution for the problem at hand.

In this study, we consider both quadrilateral and in general polygonal meshes, see Fig.7. Quadrilateral meshes are composed of $10 \times 10,20 \times 20,40 \times 40$ and $80 \times 80$ square elements. For the X-VEM, we use the stabilization in (46), where $\alpha=1$ is chosen as the scaling parameter. We generated the polygonal meshes from Voronoi tassellations by using Polymesher [48]. In order to apply essential boundary conditions, the crack is explicitly meshed over the first element $(\mathrm{AB})$, while the remaining part of the crack $(\mathrm{BC})$ is modeled by the X-VEM.

To compute the element stiffness matrix $\boldsymbol{K}_{E}$, we implement the X-VEM of Section 4 following two different strategies: topological enrichment and geometric enrichment. In the topological enrichment, graphically represented in Fig. 8a, we only enrich the node located at the singularity of the solution. The convergence rate for this problem is given by $R=\min (2 \lambda, 2 p)$, where $\lambda$ is the order of the singularity and $p$ the polynomial degree [49]. Since in our case $\lambda=1 / 2$ and $p=1$, we obtain a convergence rate $R=1$ that is non-optimal, as we anticipated in Section 3.4. In fact, this suboptimal convergence rate is also noted in enriched finite element techniques for fracture problems, cf. [50]. Figure 9 shows convergence plots of the relative error in strain energy. The expected convergence rate $R$ is reported in the graph. Both VEM and $\mathrm{X}$-VEM with topological enrichment converge in strain energy with a rate close to 1 , in agreement with theory. It turns out that the X-VEM is insensitive to the type of mesh (quadrilaterals or polygons), and the results from the X-VEM are consistently more accurate than those from standard VEM.

Many prior studies have shown that geometric enrichment, i.e., enriching all the nodes within a given radius from the singularity at the crack tip, allows the standard X-FEM for fracture problems to recover the optimal convergence rate [50, 51]. In order to establish if the proposed X-VEM can deliver the optimal convergence rate $R=2$ that is predicted by theory, we enrich all nodes that are located within a ball of radius $r_{e}=0.5$ from the origin (see Fig. $8 \mathrm{~b}$ ). 


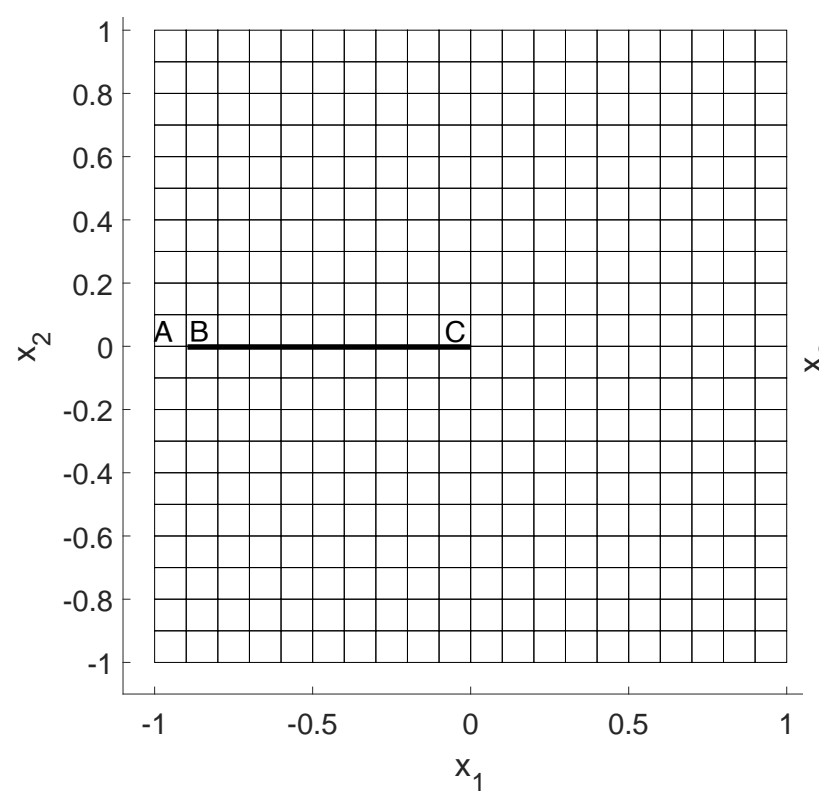

(a)

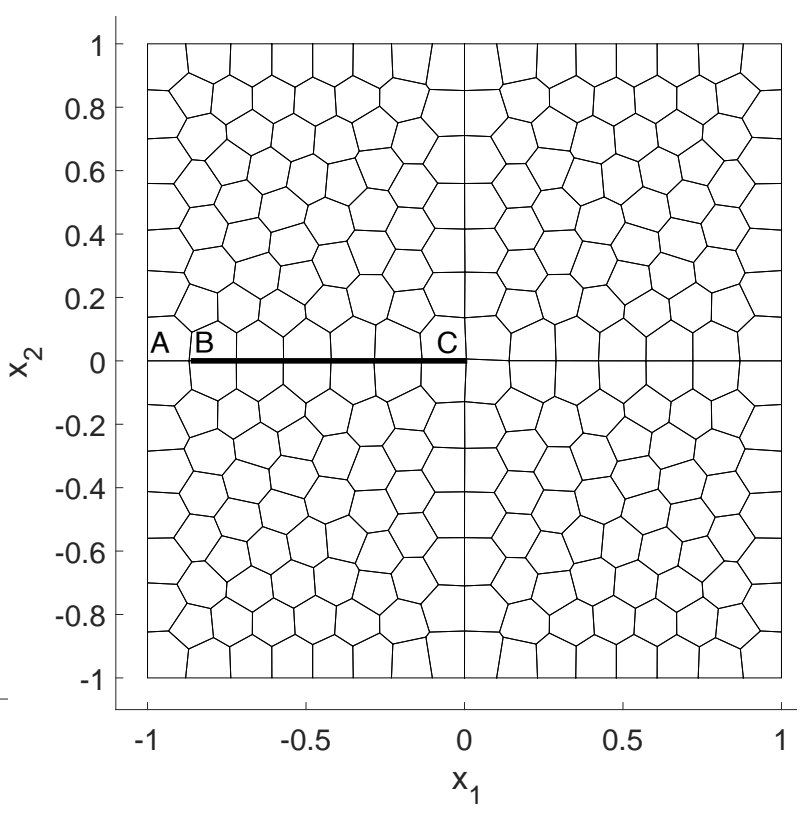

(b)

Figure 7: Mixed-mode benchmark problem. (a) Quadrilateral mesh and (b) polygonal mesh.

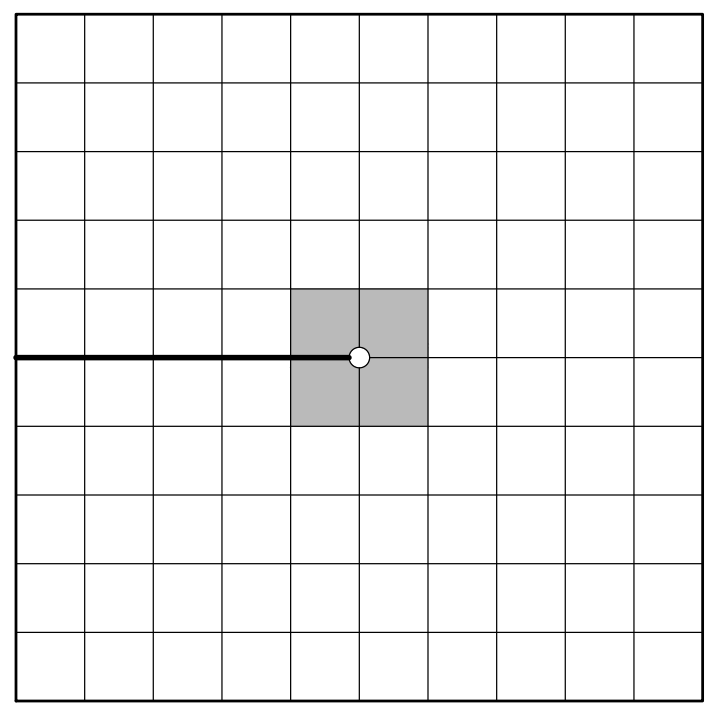

(a)

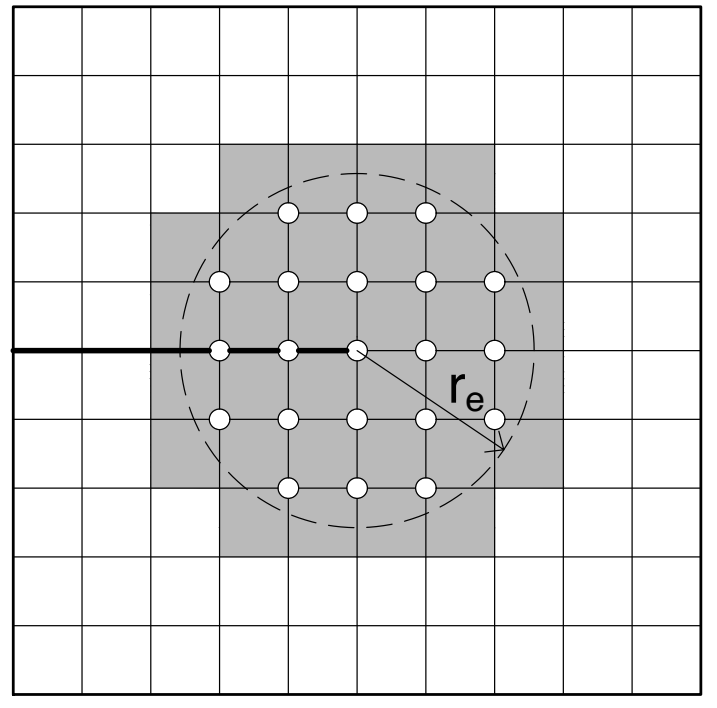

(b)

Figure 8: Mixed-mode benchmark problem. (a) Topological enrichment and (b) geometric enrichment.

Figure 10 depicts convergence plots for the relative error in strain energy on quadrilateral and polygonal meshes for the X-VEM with geometric enrichment. The convergence rate is close to 2 , which is consistent with theory. To provide a clearer picture, Fig. 11 shows a comparison between the convergence plots in strain energy for both quadrilateral and polygonal meshes.

In order to assess the robustness and the accuracy of the X-VEM in providing stress intensity factors, we apply the procedure described in Section 4.4 to the problem at hand. For this example, the exact mode I and mode II stress intensity factors are $K_{I}=1$ and $K_{I I}=1$. Both 


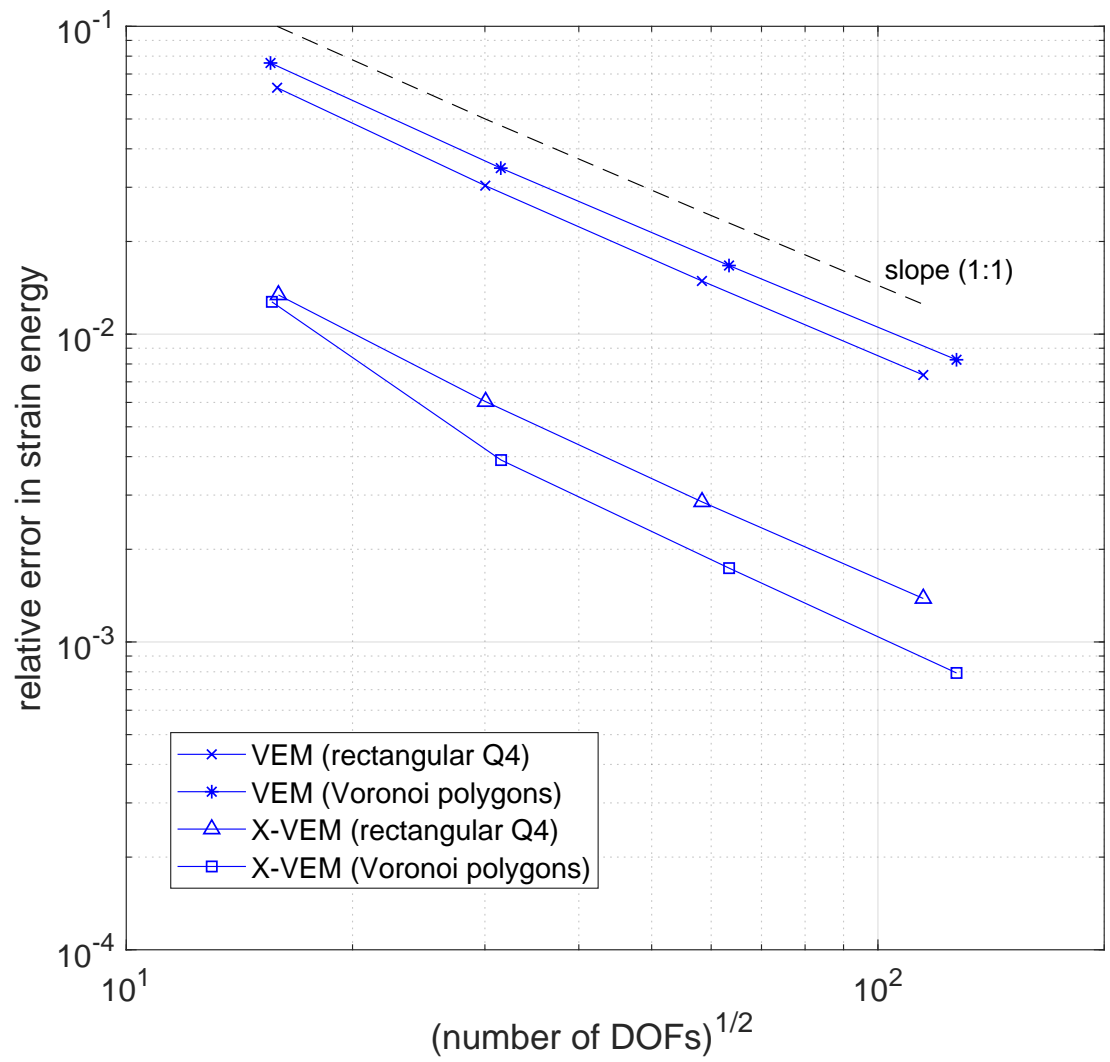

Figure 9: Convergence in strain energy for the mixed-mode benchmark problem. For the X-VEM, only the node at the origin is enriched (topological enrichment). Comparisons are shown with the standard VEM on quadrilateral and polygonal meshes. All methods converge with a rate close to unity.

topological and geometric enrichment are considered. Stress intensity factors are computed considering a ring of elements placed at a radius $r_{d}=0.4$ from the origin.

Figures $12 \mathrm{a}$ and $12 \mathrm{~b}$ show the convergence of $K_{I}$ and $K_{I I}$ on quadrilateral and polygonal meshes. Convergence is stable on all the meshes and accuracy is sound. In particular, geometric enrichment enhances both the convergence rate and the accuracy. Finally, in Fig. 13 we investigate the influence of the scaling parameter $\alpha$ in the stabilization. Convergence is optimal for $\alpha$ ranging from $10^{-3}$ to 10, with trends in improved accuracy towards smaller values of $\alpha$. Moreover, as shown in Fig. 14, $\alpha$ does not need to be adjusted if the Young modulus $E$ is varied: for a given $\alpha$, the accuracy of the method is not significantly influenced by varying $E$.

\subsection{Inclined edge crack in a finite plate under uniform tension}

We now study the problem of an inclinded edge crack in a finite plate under uniform tension. The geometry and boundary conditions are shown in Fig. 15. The plate width $W=6$ and plate height $H=3$ are chosen. The crack has length $a=1$ and is inclined at an angle $\beta$. Uniform tractions $\sigma_{t}=1$ are applied on the top edge and horizontal rollers are imposed on the bottom edge. The exact solution for this problem in the neighborhood of the crack tip is given by a linear combination of the fields (14). However, the exact solution on the whole domain is not known in closed-form.

Figures $16 \mathrm{a}$ and $16 \mathrm{~b}$ show the convergence plots (inclination angles, $\beta=0$ and $\beta=\pi / 6$ ) in 


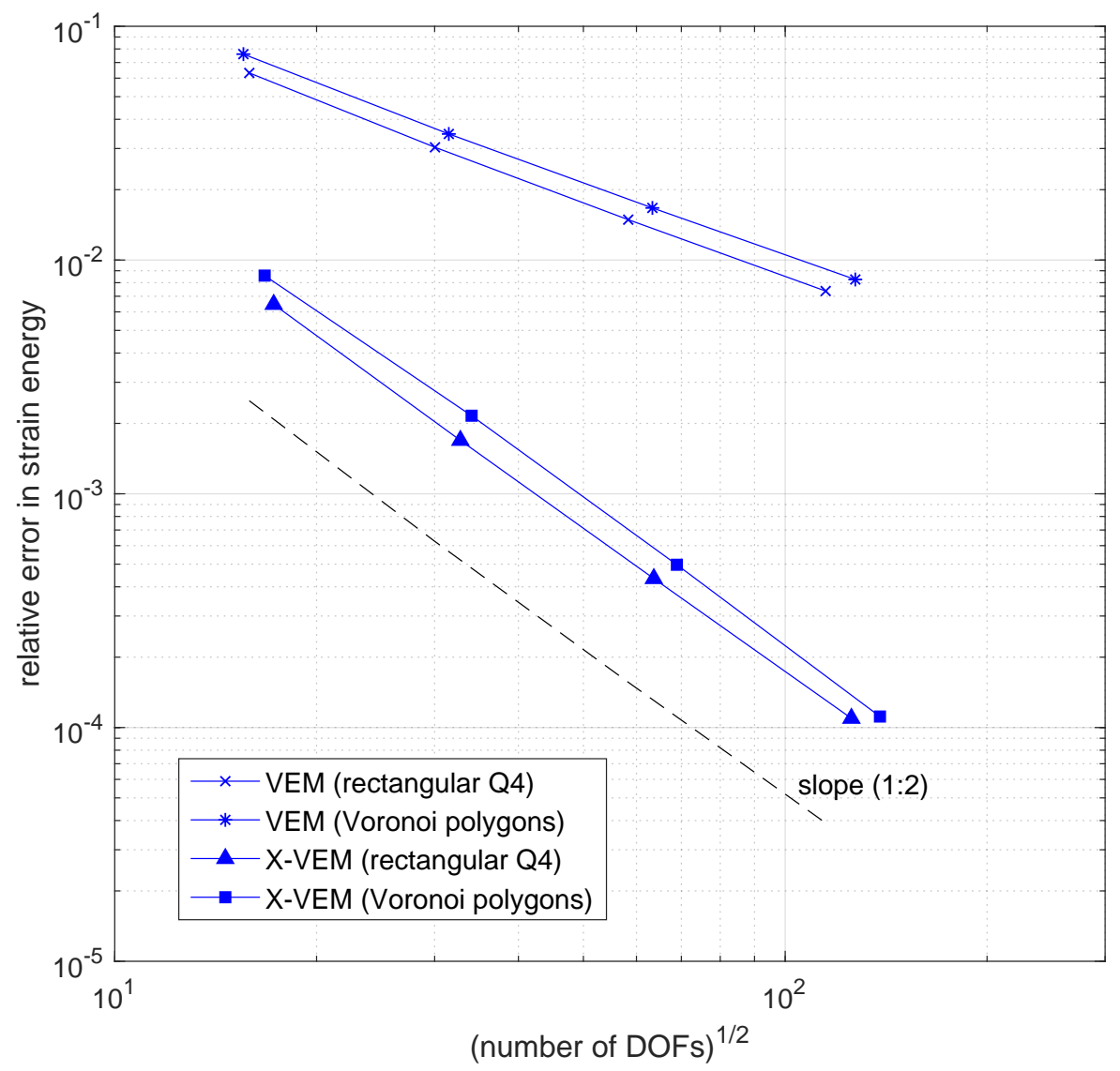

Figure 10: Convergence in strain energy for the mixed-mode benchmark problem. For the X-VEM, geometric enrichment $\left(r_{e}=0.5\right)$ on quadrilateral and polygonal meshes is used. Comparisons are made with the standard VEM. X-VEM converges with a rate close to two.

terms of the relative error in strain energy on quadrilateral and polygonal meshes. The stabilization parameter $\alpha=0.01$ is chosen. Reference solution for the energy is computed with an overkill mesh of 460.800 elements using the X-FEM. We use meshes of square elements with $h=1 / 4,1 / 10,1 / 20,1 / 40$, as well as polygonal (Voronoi) meshes. Convergence of the X-VEM is compared to standard VEM and X-FEM. The X-VEM displays sound accuracy for both mesh types, and is comparable to that obtained with the X-FEM. Finally, in Fig. 17, convergence of $K_{I}$ for $\beta=\pi / 6$ is presented for different types of meshes and enrichment radii. Again, the obtained results are in good agreement with the X-FEM, which can be inferred from Table 2, where the numerical results for $K_{I}$ and $K_{I I}$ are listed for the X-FEM and for the X-VEM with different values of the stabilization parameter $\alpha$. As already noted in Section 5.3, here too smaller values of $\alpha$ improve the accuracy of the X-VEM.

\section{Concluding remarks}

We developed a stable and convergent extended virtual element method for two-dimensional elastic fracture problems, which permits the incorporation of crack-tip singularities and discontinuities in the approximation space. Inspired by the construction of the X-FEM [3], we augmented the standard virtual element space by means of additional vectorial basis functions that 


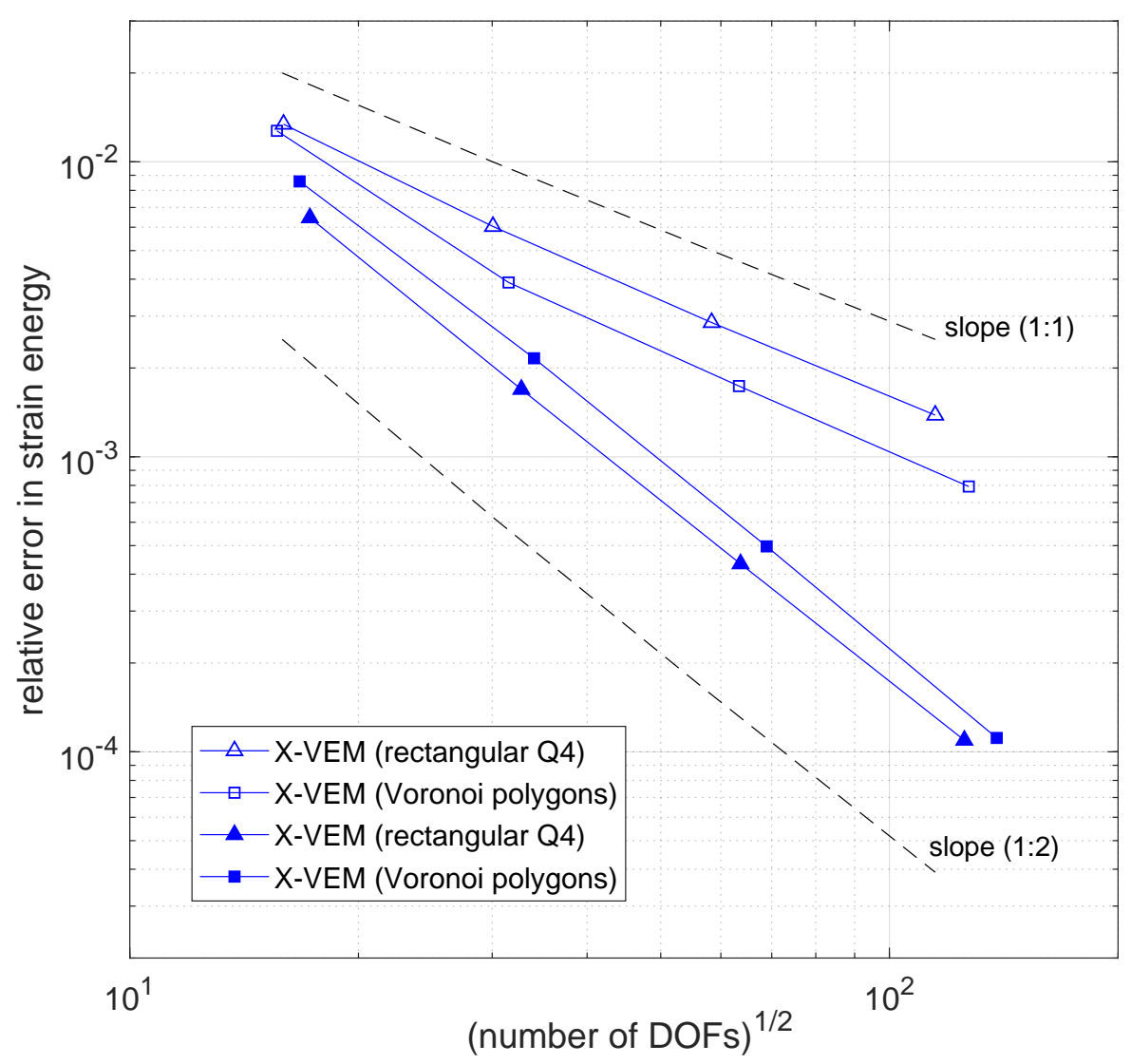

Figure 11: Convergence in strain energy for the mixed-mode benchmark problem using topological enrichment (hollow markers) and geometric enrichment with $r_{e}=0.5$ (solid markers) on quadrilateral and polygonal meshes.

were constructed using the asymptotic mode I and mode II crack-tip displacement fields as enrichment functions. An extended elliptic projector was proposed that projects the functions of the extended virtual element space onto the space spanned by linear polynomials and the enrichment fields. Crack discontinuities were modeled by decomposing each virtual shape function as the sum of two discontinuous shape functions, following the approach proposed by Hansbo and Hansbo [37]. The proposed extended virtual element formulation does not present integration issues, since all integrals are computed on the elements boundary, where virtual shape functions are known. A one-dimensional Gauss quadrature rule proved to be sufficient. On forming the element projection matrix, the consistency part of the stiffness matrix was computed and standard VEM procedures were followed to obtain the element stabilization matrix. Special attention was required to form the stabilization matrix on partially enriched elements, and an ad-hoc stabilization strategy was devised that delivered accurate results. Finally, we proposed a procedure for the computation of stress intensity factors, which entails the discretization of the annular J-domain with a ring of polygonal elements and the evaluation of the interaction integral after transforming it to a boundary integral by means of the divergence theorem.

In order to assess the consistency and the robustness of the proposed X-VEM, we conducted several numerical tests. First, we carried out an extended patch test to ensure the consistency of the method with the mode I and mode II near-tip displacement fields chosen as enrichments. We also performed a discontinuous patch test to verify the consistency of the Hansbo and Hansbo 


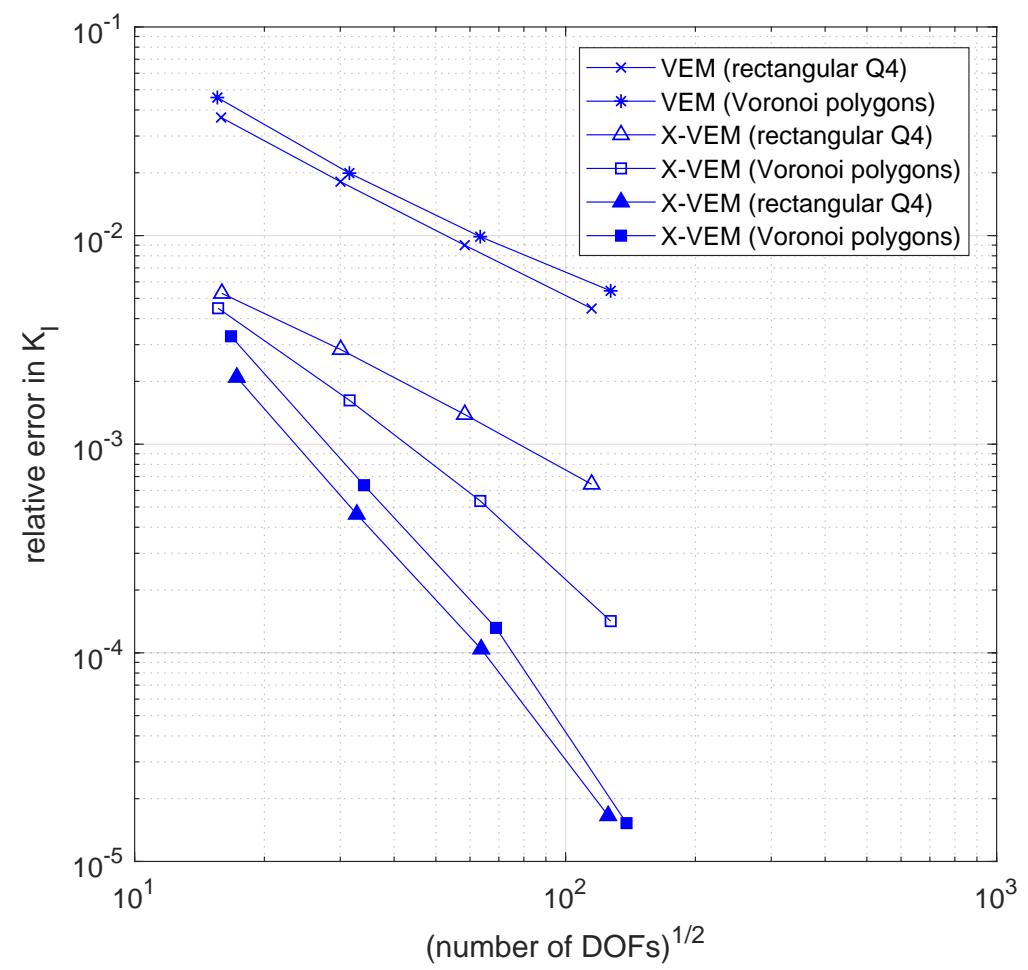

(a)

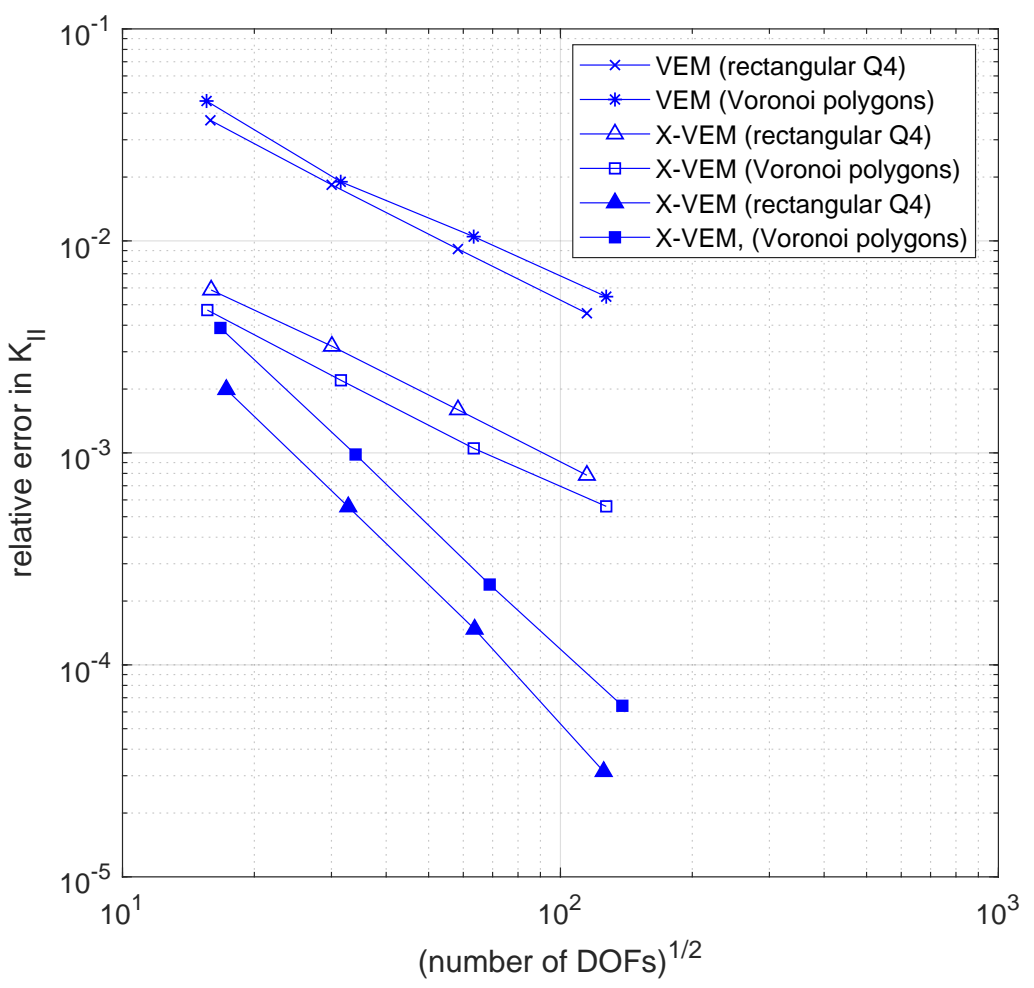

(b)

Figure 12: Convergence of stress intensity factors for the mixed-mode benchmark problem using topological enrichment (hollow markers) and geometric enrichment with $r_{e}=0.5$ (solid markers) on quadrilateral and polygonal meshes. (a) $K_{I}$ and (b) $K_{I I}$. 


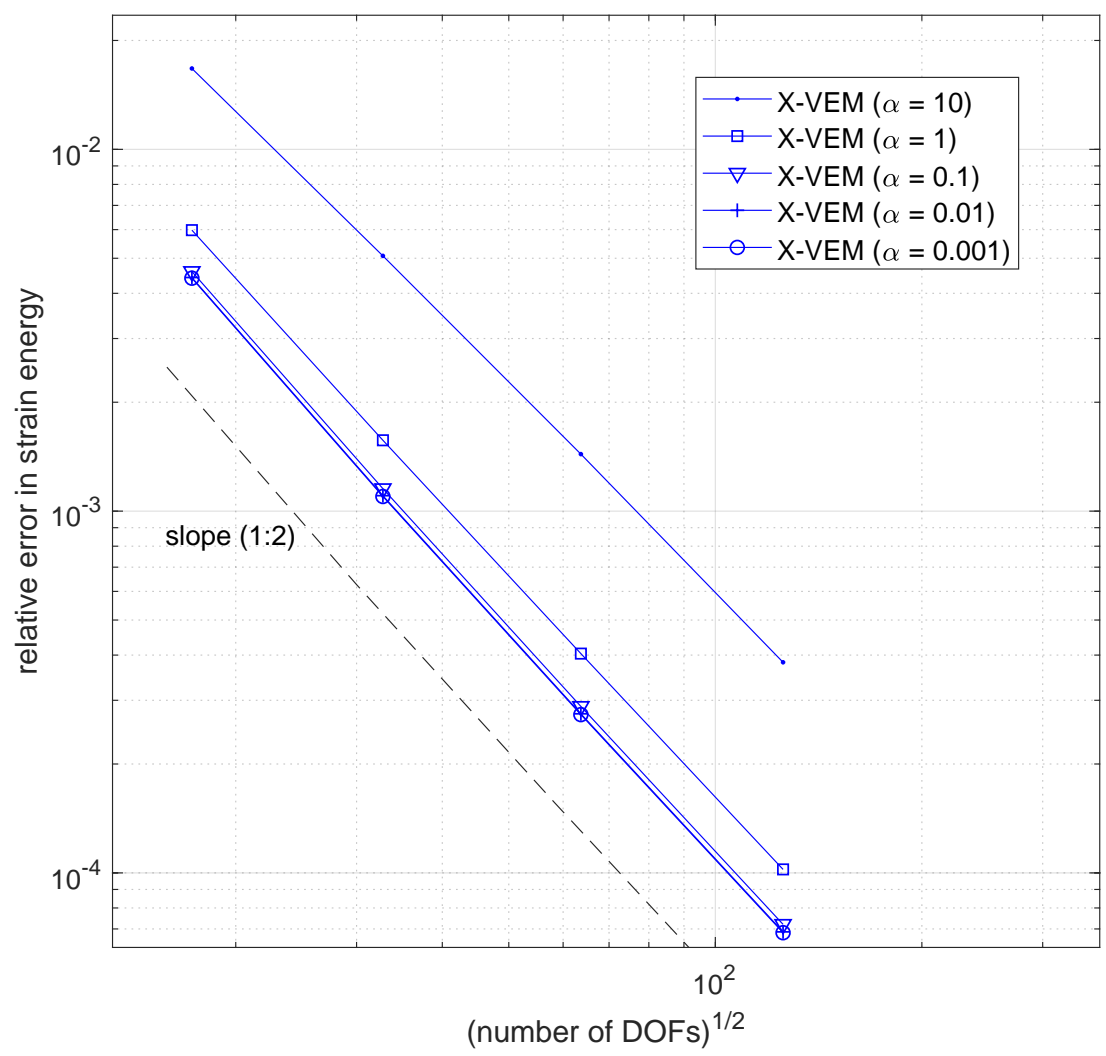

Figure 13: Convergence in strain energy for the mixed-mode benchmark problem. Convergence curves are shown for the X-VEM on a quadrilateral mesh with geometric enrichment and different choices of the stabilization parameter $\alpha$.

approach [37] that we used to incorporate the discontinuities. Then, we performed convergence studies on quadrilateral and polygonal meshes with the X-VEM on the benchmark problem of an edge crack in a square plate that is subjected to boundary conditions that are consistent with the exact mixed-mode near-tip displacement solutions. To this end, we considered topological and geometric enrichment strategies. In particular, we showed that geometric enrichment allows the method to deliver optimal convergence rates in strain energy as well as in terms of mixedmode stress intensity factors. Finally, the X-VEM was used to solve the problem of an inclined crack in a finite plate under uniform tension, and the SIF results from the X-VEM were found to be in good agreement with those computed using standard X-FEM. In future work, we will investigate the proposed extended virtual element formulation for tracking crack growth in both two- and three-dimensional elastic media.

\section{Acknowledgements}

Elena Benvenuti and Andrea Chiozzi gratefully acknowledge the support of PRIN, Italy: Progetti di Ricerca di Rilevante Interesse Nazionale (Bando 2015) Prot.2015LYYXA8. Andrea Chiozzi acknowledges the support of the research fund FIR 2020 of the University of Ferrara, Italy. Gianmarco Manzini gratefully acknowledges the financial support of the ERC Project CHANGE, which has received funding from the European Research Council under the European Union's Horizon 2020 research and innovation program (grant agreement no. 694515). 


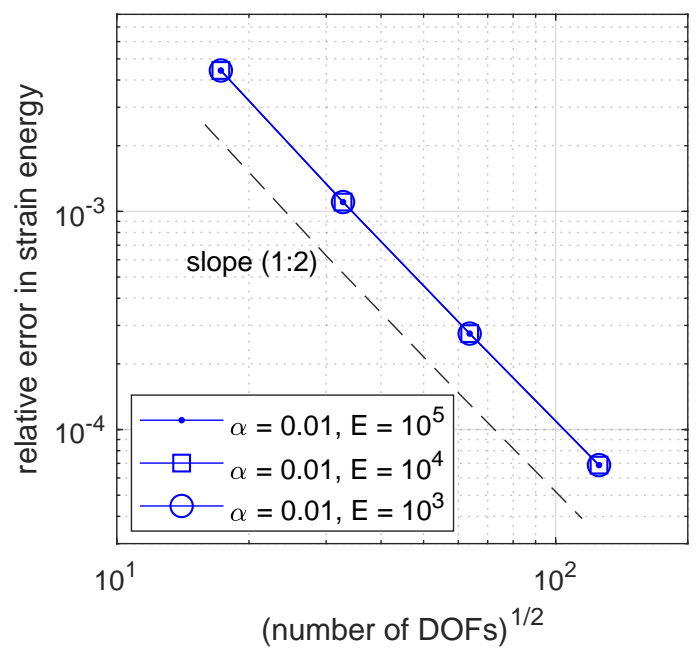

(a)

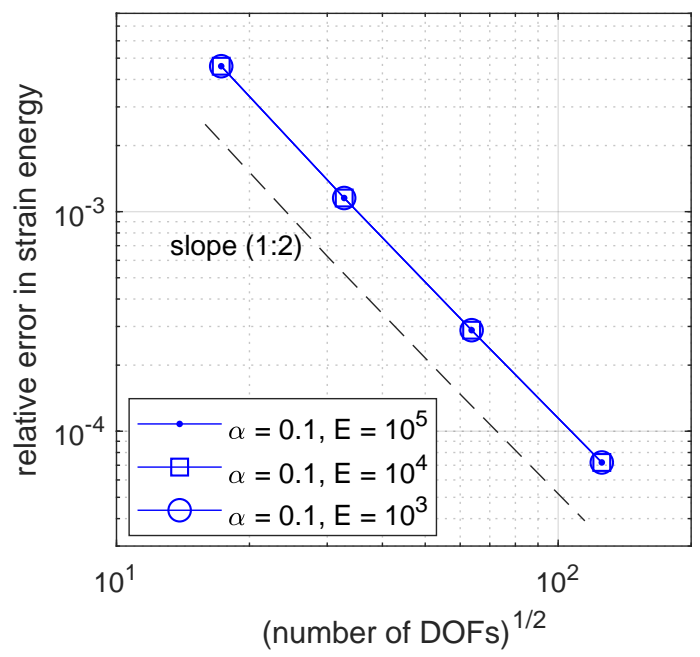

(b)

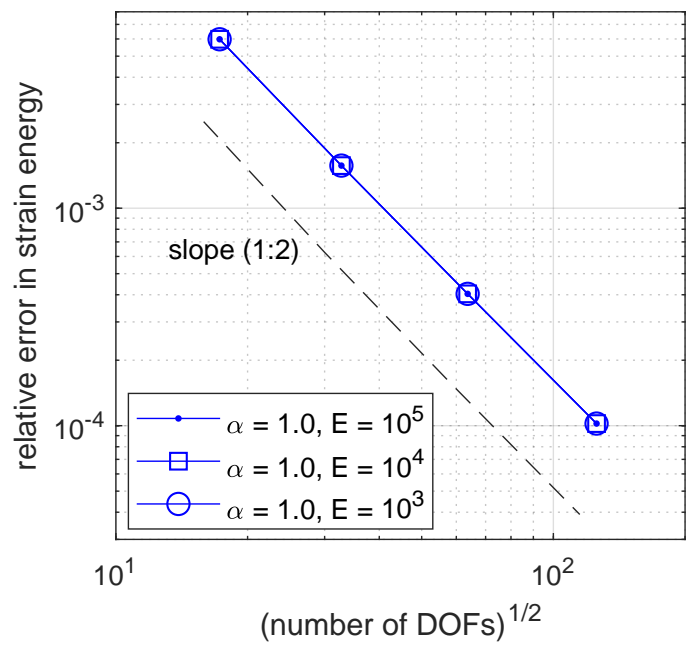

(c)

Figure 14: Convergence in strain energy for the mixed-mode benchmark problem. Convergence curves are shown for the X-VEM on a quadrilateral mesh with geometric enrichment and different choices of both the stabilization parameter $\alpha$ and the Young modulus $E$ : (a) $\alpha=0.01$, (b) $\alpha=0.1$, (c) $\alpha=1.0$ 


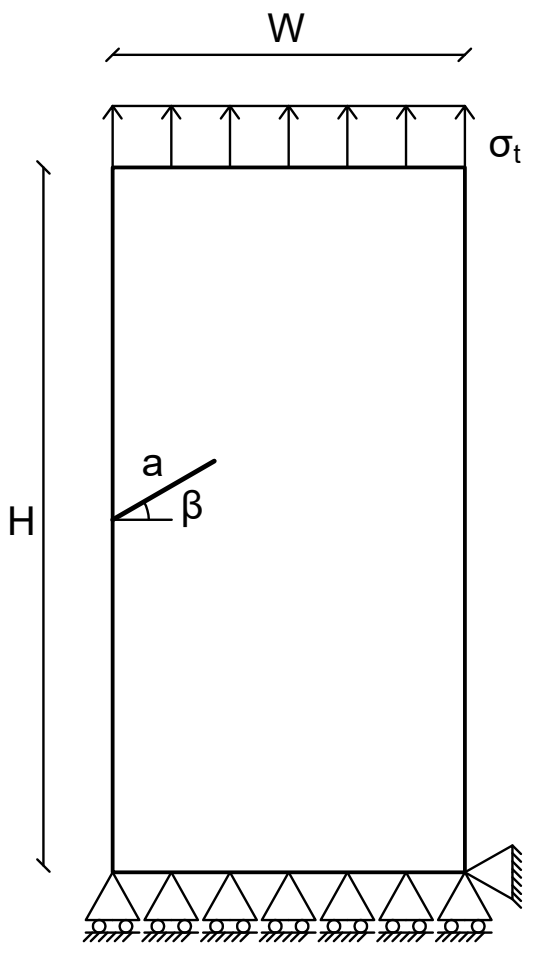

(a)

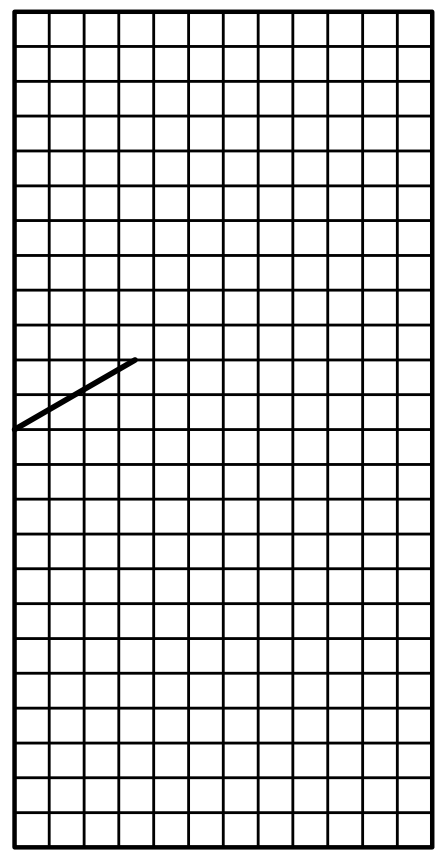

(b)

Figure 15: Inclined edge crack in a finite plate under uniform tension. (a) Problem geometry and (b) virtual element mesh.

Table 2: Stress intensity factors, $K_{I}$ and $K_{I I}$, for a finite plate with an inclined edge crack under uniform tension. Numerical results are listed for the X-FEM and the X-VEM (different values of the stabilization parameter $\alpha$ ) on a $60 \times 120$ mesh of square elements.

\begin{tabular}{ccccc|cccc}
\hline & \multicolumn{4}{c|}{$K_{I}$} & \multicolumn{3}{c}{$K_{I I}$} \\
\cline { 2 - 10 }$\beta$ & & \multicolumn{3}{c}{ X-VEM } & & X-FEM & \multicolumn{3}{c}{ X-VEM } & X-FEM \\
& $\alpha=0.01$ & $\alpha=0.05$ & $\alpha=0.10$ & & $\alpha=0.01$ & $\alpha=0.05$ & $\alpha=0.10$ \\
\hline$\pi / 12$ & 2.9351 & 2.9333 & 2.9262 & 2.9349 & 0.4631 & 0.4627 & 0.4615 & 0.4630 \\
\hline$\pi / 6$ & 2.3652 & 2.3639 & 2.3582 & 2.3651 & 0.7607 & 0.7603 & 0.7584 & 0.7606 \\
\hline$\pi / 4$ & 1.6418 & 1.6408 & 1.6370 & 1.6419 & 0.8333 & 0.8329 & 0.8308 & 0.8334 \\
\hline
\end{tabular}




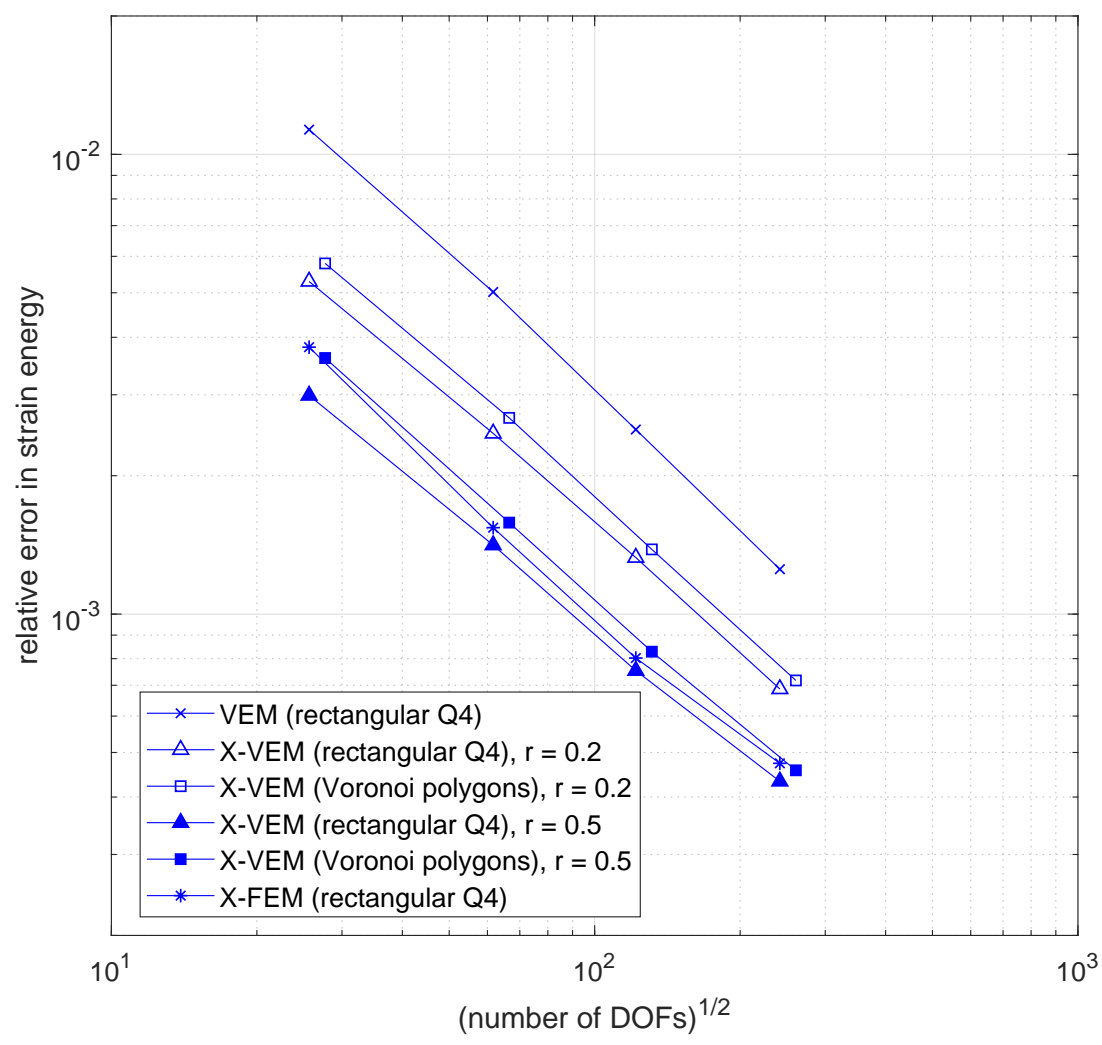

(a)

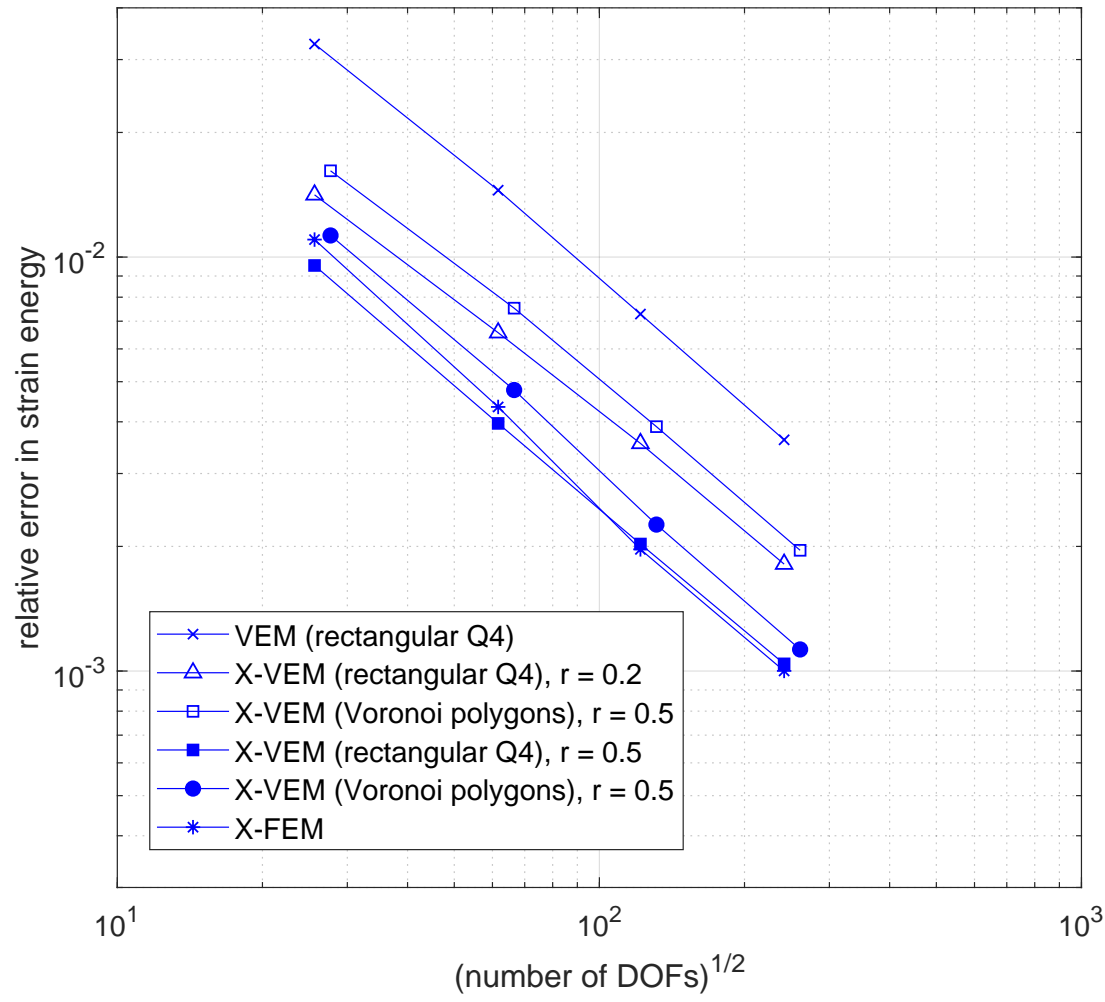

(b)

Figure 16: Convergence in strain energy for the problem of an inclined edge crack in a finite plate under uniform tension. Convergence curves are shown for quadrilateral and polygonal meshes, with varying enrichment radii. (a) $\beta=0$ and (b) $\beta=\pi / 6$. 


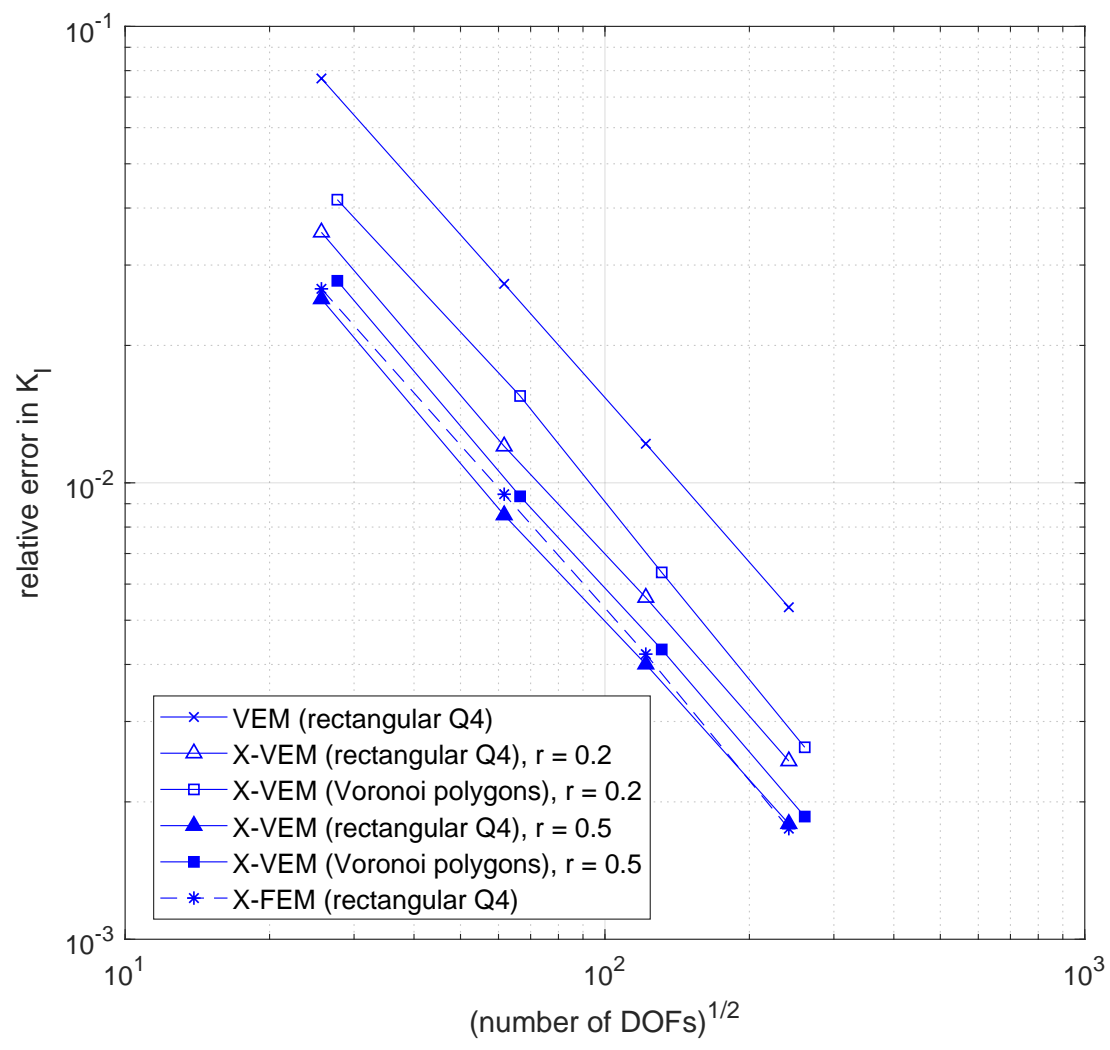

Figure 17: Convergence of $K_{I}$ for the problem of an inclined edge crack in a finite plate under uniform tension $(\beta=\pi / 6)$. Convergence curves are shown for quadrilateral and polygonal meshes, with varying enrichment radii. 


\section{References}

[1] J. M. Melenk and I. Babuška. The partition of unity finite element method: Basic theory and applications. Computer Methods in Applied Mechanics and Engineering, 139:289314, 1996.

[2] I. Babuška and J. M. Melenk. The partition of unity method. International Journal for Numerical Methods in Engineering, 40:727-758, 1997.

[3] N. Moës, J. Dolbow, and T. Belytschko. A finite element method for crack growth without remeshing. International Journal for Numerical Methods in Engineering, 46(1):131-150, 1999.

[4] A. Tabarraei and N. Sukumar. Extended finite element method on polygonal and quadtree meshes. Computer Methods in Applied Mechanics and Engineering, 197(5):425-438, 2008.

[5] A. Zamani and M. R. Eslami. Embedded interfaces by polytope FEM. International Journal for Numerical Methods in Engineering, 88:715-748, 2011.

[6] C. Song and J. P. Wolf. The scaled boundary finite-element method - alias consistent infinitesimal finite-element cell method - for elastodynamics. Computer Methods in Applied Mechanics and Engineering, 147:329-355, 1997.

[7] C. Song and J. P. Wolf. Semi-analytical representation of stress singularity as occurring in cracks in anisotropic multi-materials with the scaled boundary finite-element method. Computer and Structures, 80:183-197, 2002.

[8] C. Song, E. T. Ooi, and S. Natarajan. A review of the scaled boundary finite element method for two-dimensional linear elastic fracture mechanics. Engineering Fracture Mechanics, 187:45-73, 2018.

[9] S. E. Mousavi and N. Sukumar. Generalized Duffy transformation for integrating vertex singularities. Computational Mechanics, 45(2-3):127-140, 2010.

[10] E. B. Chin, J. B. Lasserre, and N. Sukumar. Numerical integration of homogeneous functions on convex and nonconvex polygons and polyhedra. Computational Mechanics, 56(6):967-981, 2015.

[11] E. B. Chin, J. B. Lasserre, and N. Sukumar. Modeling crack discontinuities without element-partitioning in the extended finite element method. International Journal for Numerical Methods in Engineering, 86(11):1021-1048, 2017.

[12] L. Beirão da Veiga, F. Brezzi, A. Cangiani, G. Manzini, L. D. Marini, and A. Russo. Basic principles of virtual element methods. Mathematical Models $\mathcal{F}$ Methods in Applied Sciences, 23:119-214, 2013.

[13] K. Lipnikov, G. Manzini, and M. Shashkov. Mimetic finite difference method. Journal of Computational Physics, 257(Part B.):1163-1227, 2014. 
[14] L. Beirão da Veiga, K. Lipnikov, and G. Manzini. The Mimetic Finite Difference Method, volume 11 of MSEAA. Modeling, Simulations and Applications. Springer, I edition, 2014.

[15] L. Beirão da Veiga, F. Brezzi, and D. Marini. Virtual elements for linear elasticity problems. SIAM Journal on Numerical Analysis, 51(2):794-812, 2013.

[16] A. L. Gain, C. Talischi, and G. H. Paulino. On the virtual element method for threedimensional linear elasticity problems on arbitrary polyhedral meshes. Computer Methods in Applied Mechanics and Engineering, 282:132-160, 2014.

[17] H. Chi, L. Beirão da Veiga, and G. H. Paulino. Some basic formulations of the virtual element method (vem) for finite deformations. Computer Methods in Applied Mechanics and Engineering, 318:142-190, 2017.

[18] P. Wriggers, B. D. Reddy, W. T. Rust, and B. Hudobivnik. Efficient virtual element formulations for compressible and incompressible finite deformations. Computational Mechanics, 60:253-268, 2017.

[19] K. Park, H. Chi, and G.H. Paulino. B-bar virtual element method for nearly incompressible and compressible materials. Meccanica, 56:1423-1439, 2012.

[20] L. Beirão da Veiga, C. Lovadina, and D. Mora. A virtual element method for elastic and inelastic problems on polytope meshes. Computer Methods in Applied Mechanics and Engineering, 295:327-346, 2015.

[21] B. Hudobivnik, F. Aldakheel, and P. Wriggers. A low order 3d virtual element formulation for finite elasto-plastic deformations. Computational Mechanics, 63:253—-269, 2019.

[22] E. Artioli, S. De Miranda, C. Lovadina, and L. Patruno. A stress/displacement virtual element method for plane elasticity problems. Computer Methods in Applied Mechanics and Engineering, 325:155-174, 2017.

[23] F. Dassi, C. Lovadina, and M. Visinoni. A three-dimensional Hellinger-Reissner virtual element method for linear elasticity problems. Computer Methods in Applied Mechanics and Engineering, 364:112910, 2020.

[24] E. Artioli, L. Beirão da Veiga, and F. Dassi. Curvilinear virtual elements for $2 \mathrm{~d}$ solid mechanics applications. Computer Methods in Applied Mechanics and Engineering, 359:112667, 2020.

[25] K. Park, H. Chi, and Paulino G.H. On nonconvex meshes for elastodynamics using virtual element methods with explicit time integration. International Journal for Numerical Methods in Engineering, 356:669-684, 2019.

[26] K. Park, H. Chi, and Paulino G.H. Numerical recipes for elastodynamic virtual element methods with explicit time integration. International Journal for Numerical Methods in Engineering, 121:1-31, 2020. 
[27] P. F. Antonietti, G. Manzini, I. Mazzieri, H. M. Mourad, and M. Verani. The arbitraryorder virtual element method for linear elastodynamics models: convergence, stability and dispersion-dissipation analysis. International Journal for Numerical Methods in Engineering, 122:934-971, 2021.

[28] M. F. Benedetto, S. Berrone, S. Pieraccini, and S. Scialò. The virtual element method for discrete fracture network simulations. Computer Methods in Applied Mechanics and Engineering, 280:135 - 156, 2014.

[29] V. M. Nguyen-Thanh, X. Zhuang, H. Ngyyen-Xuan, T. Rabczuk, and P. Wriggers. A Virtual Element Method for 2D linear elastic fracture analysis. Computer Methods in Applied Mechanics and Engineering, 340:366-395, 2018.

[30] A. Hussein, F. Aldakheel, B. Hudobivnik, P. Wrigger, P.A. Guidault, and O. Allix. A computational framework for brittle crack-propagation based on efficient virtual element method. Computer Methods in Applied Mechanics and Engineering, 159:15-32, 2019.

[31] E. Artioli, S. Marfia, and E. Sacco. VEM-based tracking algorithm for cohesive/frictional 2d fracture. Computer Methods in Applied Mechanics and Engineering, 365:112956, 2020.

[32] I. Perugia, P. Pietra, and A. Russo. A plane wave virtual element method for the Helmholtz problem. ESAIM: Mathematical Modelling and Numerical Analysis, 50(3):783-808, 2016.

[33] E. Benvenuti, A. Chiozzi, G. Manzini, and N. Sukumar. Extended virtual element method for the Laplace problem with singularities and discontinuities. Computer Methods in Applied Mechanics and Engineering, 356:571-597, 2019.

[34] A. Chiozzi and E. Benvenuti. Extended virtual element method for the torsion problem of cracked prismatic beams. Meccanica, 55:637-648, 2020.

[35] E. Artioli and L. Mascotto. Enrichment of the nonconforming virtual element method with singular functions. Computer Methods in Applied Mechanics and Engineering, 385:114024, 2021.

[36] C. A. Duarte, I. Babuška, and J. T. Oden. Generalized finite element methods for threedimensional structural mechanics problems. Computer and Structures, 77:215-232, 2000.

[37] A. Hansbo and P. Hansbo. A finite element method for the simulation of strong and weak discontinuities in solid mechanics. Computer Methods in Applied Mechanics and Engineering, 193(33-35):3523-3540, 2004.

[38] P. Grisvard. Singularities in Boundary Value Problems. Masson, Paris, France, 1992.

[39] P. M. A. Areias and T. Belytschko. A comment on the article 'A finite element method for simulation of strong and weak discontinuities in solid mechanics' by A. Hansbo and P. Hansbo [Comput. Methods Appl. Mech. Engrg. 193 (2004) 3523-3540]. Computer Methods in Applied Mechanics and Engineering, 195:1275-1276, 2006. 
[40] J. Duchon. Splines minimizing rotation-invariant semi-norms in Sobolev spaces. In Constructive Theory of Functions of Several Variables, volume 571 of Lecture Notes in Mathematics, pages 85-100. Springer-Verlag, Berlin, Germany, 1977.

[41] E. Benvenuti and N. Orlando. A mesh-independent framework for crack tracking in elastodamaging materials through the regularized extended finite element method. Computational Mechanics, 68:25-49, 2021.

[42] L. Beirão da Veiga, F. Brezzi, L. D. Marini, and A. Russo. The hitchhiker's guide to the virtual element method. Mathematical Models $\mathcal{F}$ Methods in Applied Sciences, 24(8):15411573, 2014.

[43] L. Beirão da Veiga, F. Dassi, and A. Russo. High-order virtual element method on polyhedral meshes. Computers and Mathematics with Applications, 74:1110-1122, 2017.

[44] J. Rice. A path independent integral and the approximate analysis of strain concentration by notches and cracks. Journal of Applied Mechanics, 35:379-386, 1968.

[45] F. Z. Li, C. F. Shih, and A. Needleman. A comparison of methods for calculating energy release rates. Engineering Fracture Mechanics, 21(2):405-421, 1985.

[46] L. Beirão da Veiga, A. Chernov, L. Mascotto, and A. Russo. Exponential convergence of the hp virtual element method in presence of corner singularities. Numerische Mathematik, 138:581-613, 2018.

[47] J. E. Dolbow and A. Devan. Enrichment of enhanced assumed strain approximations for representing strong discontinuities: addressing volumetric incompressibility and the discontinuous patch test. International Journal for Numerical Methods in Engineering, 59(1):47-67, 2004.

[48] C. Talischi, G. H. Paulino, A. Pereira, and F. M. Menezes. PolyMesher: a general-purpose mesh generator for polygonal elements written in Matlab. Structural and Multidisciplinary Optimization, 45(3):309-328, 2012.

[49] P. Grisvard. Elliptic Problems in Nonsmooth Domains. Pitman Publishing, Inc, Boston, MA, 1985.

[50] P. Laborde, J. Pommier, Y. Renard, and M. Salaün. High-order extended finite element method for cracked domains. International Journal for Numerical Methods in Engineering, 64(3):354-381, 2005.

[51] E. Béchet, H. Minnebo, N. Moës, and B. Burgardt. Improved implementation and robustness study of the X-FEM for stress analysis around cracks. International Journal for Numerical Methods in Engineering, 64(8):1033-1056, 2005. 